\title{
Protein signatures as potential surrogate biomarkers for stratification and prediction of treatment response in chronic myeloid leukemia patients
}

\author{
AYODELE A. ALAIYA ${ }^{1}$, MAHMOUD ALJURF ${ }^{4}$, ZAKIA SHINWARI ${ }^{1}$, FAHAD ALMOHAREB $^{4}$, \\ HAFIZ MALHAN $^{4}$, HAZZAA ALZAHRANI ${ }^{4}$, TAREK OWAIDAH ${ }^{3}$, JONATHAN FOX ${ }^{2}$, \\ FAHAD ALSHARIF ${ }^{4}$, SAID Y. MOHAMED $^{4}$, WALID RASHEED ${ }^{4}$, GHUZAYEL ALDAWSARI $^{4}$, \\ AMR HANBALI $^{4}$, SYED OSMAN AHMED $^{4}$ and NAEEM CHAUDHRI ${ }^{4}$
}

\begin{abstract}
${ }^{1}$ Proteomics Unit, Stem Cell and Tissue Re-Engineering Program, King Faisal Specialist Hospital and Research Centre, (KFSH\&RC), Riyadh, 11211 Saudi Arabia; ${ }^{2}$ Waters U.K. Limited, Atlas Park, Simonsway, Manchester, M22 5PP, UK; ${ }^{3}$ Hematopathology Section, Department of Pathology and Laboratory Medicine, and ${ }^{4}$ Adult Hematology/HSCT Section, Oncology Center, King Faisal Specialist Hospital and Research Centre, (KFSH\&RC), Riyadh, 11211 Saudi Arabia
\end{abstract}

Received April 8, 2016; Accepted May 4, 2016

DOI: $10.3892 /$ ijo.2016.3618

\begin{abstract}
There is unmet need for prediction of treatment response for chronic myeloid leukemia (CML) patients. The present study aims to identify disease-specific/diseaseassociated protein biomarkers detectable in bone marrow and peripheral blood for objective prediction of individual's best treatment options and prognostic monitoring of CML patients. Bone marrow plasma (BMP) and peripheral blood plasma (PBP) samples from newly-diagnosed chronic-phase CML patients were subjected to expression-proteomics using quantitative two-dimensional gel electrophoresis (2-DE) and label-free liquid chromatography tandem mass spectrometry (LC-MS/MS). Analysis of 2-DE protein fingerprints preceding therapy commencement accurately predicts 13 individuals that
\end{abstract}

Correspondence to: Dr Ayodele A. Alaiya, Proteomics Unit, Stem Cell and Tissue Re-Engineering Program, King Faisal Specialist Hospital and Research Centre, (KFSH\&RC), P.O. Box 3354, Riyadh, 11211 (MBC \#03) Saudi Arabia

E-mail: aalaiya@kfshrc.edu.sa

E-mail: maljurf@kfshrc.edu.sa

E-mail: chaudhri@kfshrc.edu.sa

Abbreviations: CML, chronic myeloid leukemia; CMR, complete molecular response; $\mathrm{CP}$, chronic phase; DAS, dasatinib; IM, imatinib mesylate; MCyR, major cytogenetic response; MMR, major molecular response; No-MMR, no-major molecular response; LT-MMR, long-term-MMR; P-No-MMR; persistent-no-MMR; TKI, tyrosine kinase inhibitors; 2-DE, two-dimensional gel electrophoresis; LC-MS/MS, liquid chromatography coupled with tandem mass spectrometry

Key words: proteomics, chronic myeloid leukemia, treatment response, biomarkers, tyrosine kinase inhibitor, imatinib achieved major molecular response (MMR) at 6 months from 12 subjects without MMR (No-MMR). Results were independently validated using LC-MS/MS analysis of BMP and PBP from patients that have more than 24 months followed-up. One hundred and sixty-four and 138 proteins with significant differential expression profiles were identified from PBP and BMP, respectively and only 54 proteins overlap between the two datasets. The protein panels also discriminates accurately patients that stay on imatinib treatment from patients ultimately needing alternative treatment. Among the identified proteins are TYRO3, a member of TAM family of receptor tyrosine kinases (RTKs), the S100A8, and MYC and all of which have been implicated in CML. Our findings indicate analyses of a panel of protein signatures is capable of objective prediction of molecular response and therapy choice for CML patients at diagnosis as 'personalized-medicine-model'.

\section{Introduction}

Chronic myeloid leukemia (CML) is unequivocally distinguishable from other myeloproliferative disorders by the presence of a reciprocal translocation of chromosomes 9 and 22 (1-3). Although the Philadelphia chromosome is detected in $90-95 \%$ of CML patients, evidence of the BCR-ABL rearrangement is also usually detected in the subgroup of Philadelphia chromosome-negative CML patients (4-6).

The presence of BCR-ABL in CML patients and the requirement of kinase activity for BCR-ABL function make this an attractive target for selective kinase inhibitors.

The old traditional therapy of newly diagnosed chronic phase-CML patients includes busulfan and hydroxyurea and most of the patients will stay in a chronic phase for approximately 3-5 years $(7,8)$. Treatment of CML later evolved to where the goal was prolongation of the chronic phase through induction of karyotypic remission and possibly molecular remission using Alfa-interferon therapy with or without cyto- 


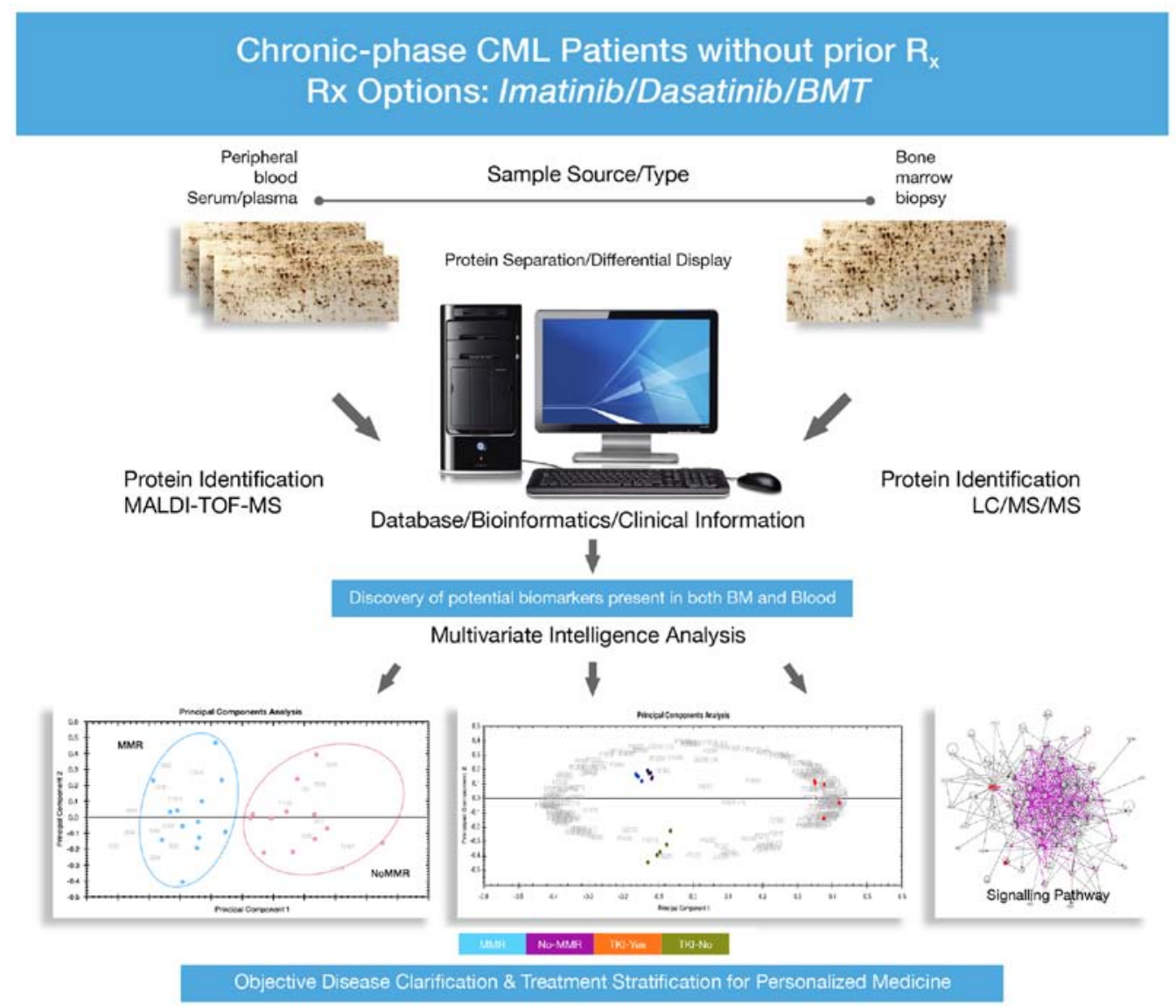

Figure 1. Overview of our biomarker discovery proteomics approach. Bone marrow and peripheral blood samples were analyzed by 2-DE and LC/MS/MS Identified proteins were subjected to statistical analysis and evaluated for early treatment response and prediction of individualized treatment options. Potential markers would be validated for clinical use.

sine arabinoside. Thereafter, imatinib mesylate (IM) a tyrosine kinase inhibitor (TKI) was introduced as potential molecular therapy for CML $(7,9)$. IM is capable of inhibiting BCR-ABL kinase activity by blocking ABL tyrosine kinase action through the binding and subsequent inactivation of the ATP-binding sites of ABL tyrosine kinase in leukemic cells $(9,10)$. Since its introduction, several clinical trials have demonstrated the efficacy of IM and new generation TKIs in the treatment of CML, including patients with interferon-refractory CP-CML, as well as patients with CML in blast crisis (11).

Approximately more than $50 \%$ of CML patients treated with imatinib achieve a complete cytogenetic response $(11,12)$. CML progression while on imatinib is usually due to the emergence of imatinib-resistant BCR-ABL mutant cells.

The relatively unpredictable biological behavior is a major challenge in its management as the chronic phase of CML is less aggressive and has very favorable prognosis with an excellent 5-year survival rate. By contrast, the biologically aggressive blast phase of CML is often rapidly fatal (2). Currently, there is no recognized prognostic value for the baseline BCR-ABL level, furthermore, there are variations in sensitivity or dependability of RQ-PCR assays across different laboratories (13). There is therefore a need to develop molecular markers for selection of choice of therapy at the time of diagnosis and to identify patients that are more likely to achieve a sustained remission, and patients who are more likely to develop resistance to imatinib therapy.
New analytical tools in proteomics are emerging that give new insights into biological processes that may speed up the discovery of potential biomarkers. Quantitative molecular variations may be used for the development of methods for tumor classification based on large amounts of gene expression data generated by 2-DE analysis of proteins $(14,15)$.

The main aim of the present study is towards discovery of objective markers that predict patients' response status and selection of appropriate choice of therapy at the onset of disease diagnosis. It focuses on the analysis of global peripheral blood plasma and bone marrow plasma protein expression profiles among CP-CML patients who achieved LT-MMR on imatinib compared with patients without MMR as well as whether or not they remain on TKI or switch to second generation TKI or requiring alternative therapy.

The endpoint is to identify disease-specific/disease-associated protein biomarkers seen in bone marrow tissue as well as in peripheral blood plasma. This would subsequently allow monitoring of such biomarker proteins in peripheral blood, rather than bone marrow, demanding less invasive procedures for objective prediction of individual's best treatment options and prognostic monitoring of CML patients.

\section{Materials and methods}

All bone marrow samples were obtained by aspiration procedure via posterior iliac crest under local anesthesia. Because 
Table I. Clinical characteristics of analyzed samples.

\begin{tabular}{|c|c|c|c|c|c|c|c|c|c|c|c|c|}
\hline \multirow[b]{2}{*}{ Samples } & \multirow[b]{2}{*}{ Gender } & \multirow[b]{2}{*}{ Age (years) } & \multicolumn{2}{|c|}{ TKI-fail } & \multicolumn{2}{|c|}{$\begin{array}{l}\text { MMR at } \\
6 \text { months }\end{array}$} & \multicolumn{2}{|c|}{$\begin{array}{c}\text { MMR at } \\
12 \text { months }\end{array}$} & \multicolumn{2}{|c|}{$\begin{array}{c}\text { MMR at } \\
18 \text { months }\end{array}$} & \multicolumn{2}{|c|}{$\begin{array}{c}\text { MMR at } \\
24 \text { months }\end{array}$} \\
\hline & & & No & Yes & No & Yes & No & Yes & No & Yes & No & Yes \\
\hline CML1 & Female & 14 & $\sqrt{ }$ & & & $\sqrt{ }$ & & $\sqrt{ }$ & & $\sqrt{ }$ & & $\sqrt{ }$ \\
\hline CML2 & Female & 14 & $\sqrt{ }$ & & & $\sqrt{ }$ & & $\sqrt{ }$ & & $\sqrt{ }$ & & $\sqrt{ }$ \\
\hline CML3 & Female & 26 & & $\sqrt{ }$ & $\sqrt{ }$ & & $\sqrt{ }$ & & & & & \\
\hline CML4 & Male & 18 & & $\sqrt{ }$ & & $\sqrt{ }$ & $\sqrt{ }$ & & $\sqrt{ }$ & & & $\sqrt{ }$ \\
\hline CML5 & Male & 50 & $\sqrt{ }$ & & & $\sqrt{ }$ & & $\sqrt{ }$ & & $\sqrt{ }$ & & $\sqrt{ }$ \\
\hline CML6 & Female & 50 & $\sqrt{ }$ & & & $\sqrt{ }$ & & $\sqrt{ }$ & & $\sqrt{ }$ & & $\sqrt{ }$ \\
\hline CML7 & Male & 41 & & $\sqrt{ }$ & & $\sqrt{ }$ & & $\sqrt{ }$ & & $\sqrt{ }$ & & $\sqrt{ }$ \\
\hline CML8 & Female & 64 & & $\sqrt{ }$ & $\sqrt{ }$ & & $\sqrt{ }$ & & $\sqrt{ }$ & & $\sqrt{ }$ & \\
\hline CML10 & Male & 27 & $\sqrt{ }$ & & & $\sqrt{ }$ & $\sqrt{ }$ & & & $\sqrt{ }$ & & $\sqrt{ }$ \\
\hline CML13 & Male & 44 & $\sqrt{ }$ & & & $\sqrt{ }$ & & $\sqrt{ }$ & & $\sqrt{ }$ & & $\sqrt{ }$ \\
\hline CML15 & Male & 21 & $\sqrt{ }$ & & & $\sqrt{ }$ & & $\sqrt{ }$ & & $\sqrt{ }$ & & $\sqrt{ }$ \\
\hline CML16 & Male & 44 & $\sqrt{ }$ & & & $\sqrt{ }$ & & $\sqrt{ }$ & & $\sqrt{ }$ & & $\sqrt{ }$ \\
\hline CML17 & Female & 18 & & $\sqrt{ }$ & $\sqrt{ }$ & & $\sqrt{ }$ & & $\sqrt{ }$ & & $\sqrt{ }$ & \\
\hline CML18 & Female & 65 & $\sqrt{ }$ & & $\sqrt{ }$ & & $\sqrt{ }$ & & $\sqrt{ }$ & & $\sqrt{ }$ & \\
\hline CML19 & Male & 26 & & $\sqrt{ }$ & $\sqrt{ }$ & & $\sqrt{ }$ & & $\sqrt{ }$ & & $\sqrt{ }$ & \\
\hline CML21 & Male & 39 & & $\sqrt{ }$ & $\sqrt{ }$ & & $\sqrt{ }$ & & & $\sqrt{ }$ & & $\sqrt{ }$ \\
\hline CML22 & Female & 67 & $\sqrt{ }$ & & & $\sqrt{ }$ & & $\sqrt{ }$ & & $\sqrt{ }$ & & $\sqrt{ }$ \\
\hline CML23 & Male & 47 & $\sqrt{ }$ & & & $\sqrt{ }$ & & $\sqrt{ }$ & & $\sqrt{ }$ & & $\sqrt{ }$ \\
\hline CML24 & Male & 18 & & $\sqrt{ }$ & $\sqrt{ }$ & & $\sqrt{ }$ & & $\sqrt{ }$ & & $\sqrt{ }$ & \\
\hline CML25 & Male & 40 & $\sqrt{ }$ & & & $\sqrt{ }$ & & $\sqrt{ }$ & & $\sqrt{ }$ & & $\sqrt{ }$ \\
\hline CML26 & Female & 30 & & $\sqrt{ }$ & $\sqrt{ }$ & & & & & & & \\
\hline CML27 & Female & 36 & $\sqrt{ }$ & & $\sqrt{ }$ & & & $\sqrt{ }$ & & $\sqrt{ }$ & & $\sqrt{ }$ \\
\hline CML28 & Female & 37 & $\sqrt{ }$ & & & $\sqrt{ }$ & & $\sqrt{ }$ & & $\sqrt{ }$ & & $\sqrt{ }$ \\
\hline CML29 & Female & 33 & & $\sqrt{ }$ & $\sqrt{ }$ & & $\sqrt{ }$ & & $\sqrt{ }$ & & & $\sqrt{ }$ \\
\hline CML30 & Female & 44 & $\sqrt{ }$ & & & $\sqrt{ }$ & & $\sqrt{ }$ & & $\sqrt{ }$ & & $\sqrt{ }$ \\
\hline CML31 & Female & 48 & & & & & & & & & & \\
\hline CML32 & Female & 38 & & $\sqrt{ }$ & $\sqrt{ }$ & & & & & & & \\
\hline CML33 & Female & 32 & & $\sqrt{ }$ & $\sqrt{ }$ & & $\sqrt{ }$ & & & & & \\
\hline CML34 & Male & 52 & & $\sqrt{ }$ & $\sqrt{ }$ & & $\sqrt{ }$ & & $\sqrt{ }$ & & $\sqrt{ }$ & \\
\hline CML38 & Male & 37 & & & & & & & & & & \\
\hline CML40 & Male & 61 & & $\sqrt{ }$ & $\sqrt{ }$ & & $\sqrt{ }$ & & $\sqrt{ }$ & & & \\
\hline CML41 & Male & 47 & $\sqrt{ }$ & & & $\sqrt{ }$ & & $\sqrt{ }$ & & $\sqrt{ }$ & & $\sqrt{ }$ \\
\hline CML43 & Female & 51 & & $\sqrt{ }$ & $\sqrt{ }$ & & $\sqrt{ }$ & & $\sqrt{ }$ & & $\sqrt{ }$ & \\
\hline CML44 & Female & 14 & $\sqrt{ }$ & & & $\sqrt{ }$ & & $\sqrt{ }$ & & $\sqrt{ }$ & & $\sqrt{ }$ \\
\hline CML45 & Female & 45 & $\sqrt{ }$ & & & $\sqrt{ }$ & & $\sqrt{ }$ & & $\sqrt{ }$ & & $\sqrt{ }$ \\
\hline CML46 & Female & 45 & $\sqrt{ }$ & & $\sqrt{ }$ & & $\sqrt{ }$ & & & $\sqrt{ }$ & & $\sqrt{ }$ \\
\hline CML47 & Male & 32 & & $\sqrt{ }$ & $\sqrt{ }$ & & $\sqrt{ }$ & & $\sqrt{ }$ & & $\sqrt{ }$ & \\
\hline Total & & & 19 & 16 & 17 & 18 & 16 & 17 & 11 & 20 & 8 & 22 \\
\hline
\end{tabular}

of limited amount of materials for analysis, the cells were not flow cytometry sorted, rather unsorted bone marrows as well as unsorted peripheral blood plasma were collected and prepared for analysis.

Bone marrow and plasma, samples obtained at diagnosis and prior to initiation of treatment from 37 patients with newly diagnosed CP-CML were subjected to expression proteome analysis using combined gel-based 2-DE and label-free in-solution quantitative liquid chromatography coupled with tandem mass spectrometry (LC-MS/MS). Patients selections into those that achieved or did not achieve MMR was based on patients with serial positive or negative responses to treatment at different time-points $(3,6$, 12 and 24 months, respectively). Patients that responded at a time-point but failed to respond at the next time-point were not included in the analysis. However, patients that did not achieve MMR at 3 months, but subsequently achieved MMR at 6, 12 and 24 months were included. Because there was fewer number of patients with MMR at 3 months, the focus of our analyzed time-points were at 6,12 and 24 months. Twenty-five patients consisting 13 with major molecular response and 12 without major molecular response were analyzed. In addition, patients that failed tyrosine kinase inhibitor (TKI) were analyzed. Four additional patients 
samples not included in the proteomics analysis were used in the western blot analysis. The overview of experimental design is shown in Fig. 1 and the clinical characteristics of all patients were as indicated in Table I.

Sample preparation protocols for proteomic analysis. All the patients with primary diagnosis of CML were recruited in Oncology Center at KFSH\&RC. From each of the patients, $10 \mathrm{ml}$ of peripheral EDTA-anti-coagulated blood (plasma) was taken. Where possible, bone marrow aspirations were obtained from the same patients in addition to peripheral blood samples.

All samples were subjected to extensive pre-analysis cleanup using human albumin removal protocols (Agilent Technologies). Written and signed informed consents were obtained from all patients and the Institution's Research Advisory Council, under the Office of Research Affairs, approved the study (RAC\# 2050-040).

Protein separation by high resolution two dimensional gel electrophoresis, (2-DE) scanning and image analysis. Equivalent amount of $50 \mathrm{mg}$ total proteins for each analyzed sample was dissolved in $350 \mathrm{ml}$ volume of rehydration buffer [2\% (v/v) IPG-buffer 4-7 linear] and loaded onto an 11-cm IPG-strip 4-7 linear (Bio-Rad Laboratories). This gave better overview of gel separated protein spots across the entire chosen $\mathrm{pH}$ window and gel images were visualized by SYPRO Ruby fluorescent staining. Stained gels were scanned using a Typhoon Trio Imager (GE) and data were analyzed using the Progenesis SameSpots software (version 7.1.0; Nonlinear Dynamics, Ltd., Newcastle, UK). Gel images were compared for qualitative and quantitative differences. In addition, the protein expression profiles were used to assess the level of individual variability and only samples with similar phenotypic changes were used for sample pools for LC/MS/MS (due to low through-put analysis) as detailed below. Polypeptide quantities were calculated based on the normalized total integrated density volume.

Protein in solution-digestion. The plasma samples were diluted and protein concentrations of all samples were normalized as previously described (16). Briefly, for analytical runs, equal amount of protein was taken from each sample to generate a pool of patient as one group. The samples within same sample cohort were pooled due to low through-put of LC/MS/MS analysis platform. However, samples were initially screened using 2-DE for homogeneity within the same analysis group. For each analysis sample group, $200 \mu \mathrm{g}$ complex protein mixture was taken and exchanged twice with $500 \mu \mathrm{l}$ of $0.1 \%$ RapiGest (Waters Corp., Manchester, UK). Protein concentrations of between 0.50 and $1 \mu \mathrm{g} / \mu \mathrm{l}$ was achieved at the end of digestion. Details of digestion protocols are as previously described $(16,17)$. Briefly, proteins were denatured in $0.1 \%$ RapiGest SF at $80^{\circ} \mathrm{C}$ for $15 \mathrm{~min}$, reduced in $10 \mathrm{mM} \mathrm{DTT}$ at $60^{\circ} \mathrm{C}$ for $30 \mathrm{~min}$, and alkylated in $10 \mathrm{mM}$ Iodoacetamide (IAA) for $40 \mathrm{~min}$ at room temperature in the dark. Samples were trypsin digested at $37^{\circ} \mathrm{C}$ overnight. Samples were diluted with aqueous $0.1 \%$ formic acid prior to LC/MS analysis in order to achieve a load of $\sim 2 \mu \mathrm{g}$ on analytical column. All samples were spiked with yeast alcohol dehydrogenase (ADH; P00330) as internal standard to the digests in order for absolute quantitation.
Protein identification by mass spectrometry: LC-MSE analysis. The digested peptides were subjected to 1-Dimensional Nano Acquity liquid chromatography coupled with tandem mass spectrometry on Synapt G2 (Waters Corp.). Expression proteomics data were generated between sample groups using both qualitative and quantitative protein changes. The ESI-MS analysis and instrument settings were optimized on the tune page as previously reported (16).

A total of $2 \mu \mathrm{l}$ sample injection representing $\sim 1 \mu \mathrm{g}$ protein digests was loaded on-column and samples were infused using the Acquity sample manager with mobile phase consisting of A1 $99 \%$ water $+1 \%$ acetonitrile $+0.1 \%$ formic acid and B1 acetonitrile $+0.1 \%$ formic acid with sample flow rate of $0.450 \mu \mathrm{l} / \mathrm{min}$. Data acquisition using iron mobility separation experiments (HDMSE) were performed and data were acquired over a range of $\mathrm{m} / \mathrm{z}$ 50-2000 Da with a total acquisition time of $115 \mathrm{~min}$. All samples were analyzed in triplicate runs (triplicate runs were repeated on two different occasions as a measure of reproducibility) and data were acquired using the MassLynx programs (version. 4.1, SCN833; Waters) operated in resolution and positive polarity modes. ProteinLynx Global Server (PLGS) 2.2 and Progenesis QI for proteomics (Progenesis QIfp version 2.0.5387) (Nonlinear Dynamics/ Waters) were used for all automated data processing and database searching. The generated peptide masses were searched against two-unified non-redundant databases (Uniprot/SwissProt Human protein sequence database) using the PLGS 2.5 and Progenesis QIfp for protein identification (Waters).

Data analysis and informatics. Progenesis QI v.2.0.5387 for proteomics was used to process and search the data to accurately quantify and identify proteins that are significantly changing between sample groups. The human database containing thousands of reviewed non-redundant entries were downloaded from UniProt/Swiss-Prot and search algorithm was applied as previoudly described (18). The criteria used for the database search were as previously described (16). Normalized label-free quantification was achieved using Progenesis QI software. The generated differentially expressed data was filtered to show only statistically (ANOVA), significantly regulated proteins $(P \leq 0.05)$ and a fold change $>1.5$. In addition, 'Hi3' absolute quantification was performed using $\mathrm{ADH}$ as an internal standard to give an absolute amount of each identified protein. These options are available as incorporated into the Progenesis QIfp (Nonlinear Dynamics/Waters).

\section{Results}

Changes in protein expression between patients with/without major molecular response at 6 months. A total of 73 protein spots on 2-DE gels differed significantly between patients that achieved MMR from those who did not achieve MMR ( $\mathrm{P}<0.05$ and at least 1.5 -fold difference). The locations of these protein spots are shown as marked on a representative 2-DE map derived from a sample with MMR in Fig. 2A. Even though the identifications of these protein spots were not done, their quantitative expression fingerprints from 2-DE analysis pattern accurately predicts 13 individuals that achieved MMR at 6 months from 12 subjects without MMR (No-MMR) using principal component analysis (PCA) (Fig. 2B). 

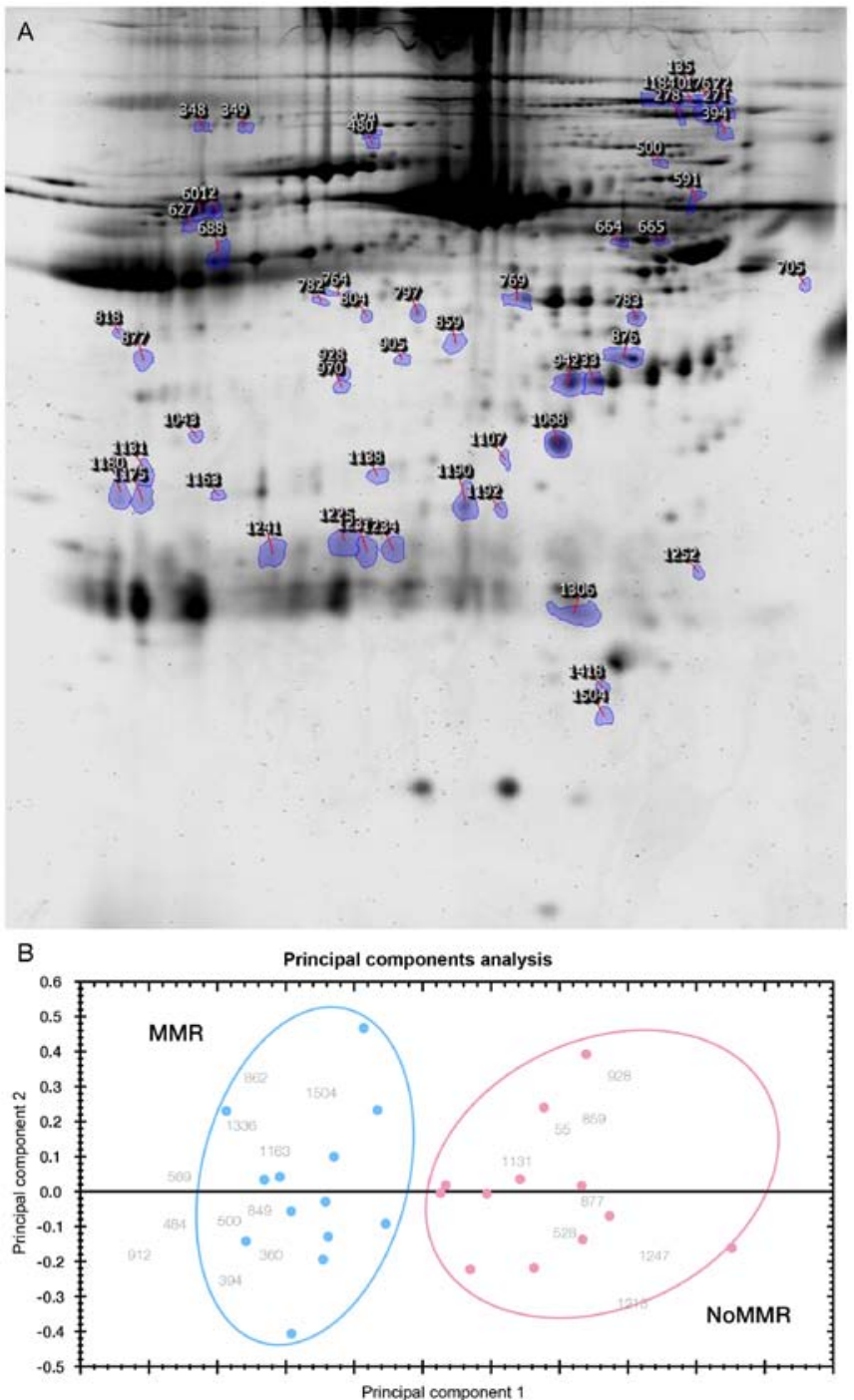

Figure 2. (A) Representative high resolution two-dimensional gel electrophoresis (2-DE) of proteins derived from CML bone marrow sample (Marked are differentially expressed protein spots between patients that achieved major molecular response from patients without major molecular response); $\mathrm{P}<0.05$ and at least 1.5 -fold difference. (B) Principal component analysis (PCA) using datasets of 73 differentially expressed protein spots between groups of CML samples based on MMR (blue) and No-MMR (pink) at 6 months. The letters in grey in the background represents the protein spot numbers on the 2-DE gel of all the implicated protein spots used in the analysis.

These findings are similar to what was observed with PCA plot generated from non-gel LC/MS/MS analysis platform, as some of the results were independently validated using the label free quantitative liquid chromatography tandem mass spectrometry as detailed below.

LC/MS/MS analysis of peripheral blood for prognostic monitoring of early CML treatment response. Peripheral blood samples were evaluated for early treatment response at 6 month and prediction of treatment options towards personalized medicine. Approximately 115 protein species were identified, of which only 64 were significantly differentially expressed between MMR and No-MMR sample groups. ( $>1.5$ - to $\infty$-fold change, $\mathrm{p}<0.05$ ). These proteins predict accurately patients with MMR vs. No-MMR

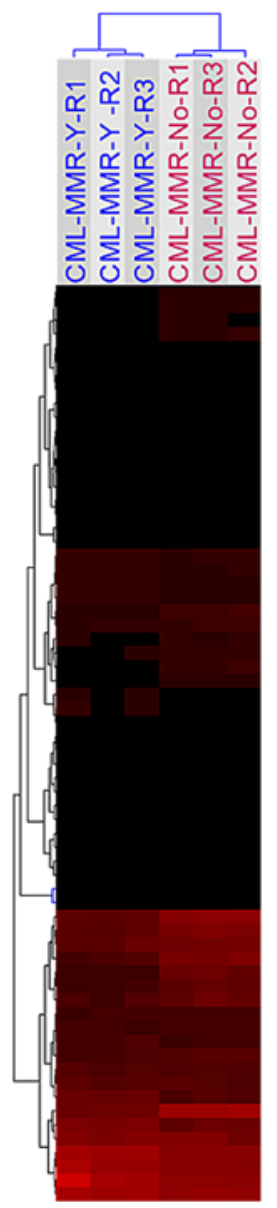

Figure 3. Unsupervised hierarchical cluster analysis of 64 identified differentially expressed proteins between patients that achieved MMR (blue) at 6 months from patients without MMR (No-MMR, red). The image was generated using J-Express Pro V 1.1 software program. (These 64 proteins used in generating this dendrogram plot are indicated by letter $\mathrm{b}$ in Table II).

patients using unsupervised Hierarchical Cluster Analysis (Fig. 3).

Evaluation of bone marrow and peripheral blood protein profiles for prognostic monitoring of prolonged and sustained treatment response vs. persistent no-major molecular response. Some of the patients have been followed for more than 24 months. Patients who have been consistent over a long-term in achieving and maintaining MMR from 6 months until 24 months were labeled as LT-MMR, while patients that have been persistent with No-MMR from 6 months until 24 months were called P-No-MMR. We believe that the ability to select early responders from 6 months all through 24 months would be very helpful to identify markers that would accurately predict patients with risk of delayed or suboptimal response further than 6 months. These cohorts of patients were considered as important in an effort to provide the possibility to identify surrogate biomarkers to evaluate long-term treatment response and discovery of disease-specific/disease-associated proteins for objective prognostic monitoring of CML patients.

Equal amounts of total peripheral blood plasma proteins from 10 LT-MMR patients were pooled and compared for their protein expressions among 10 other samples from P-No-MMR 

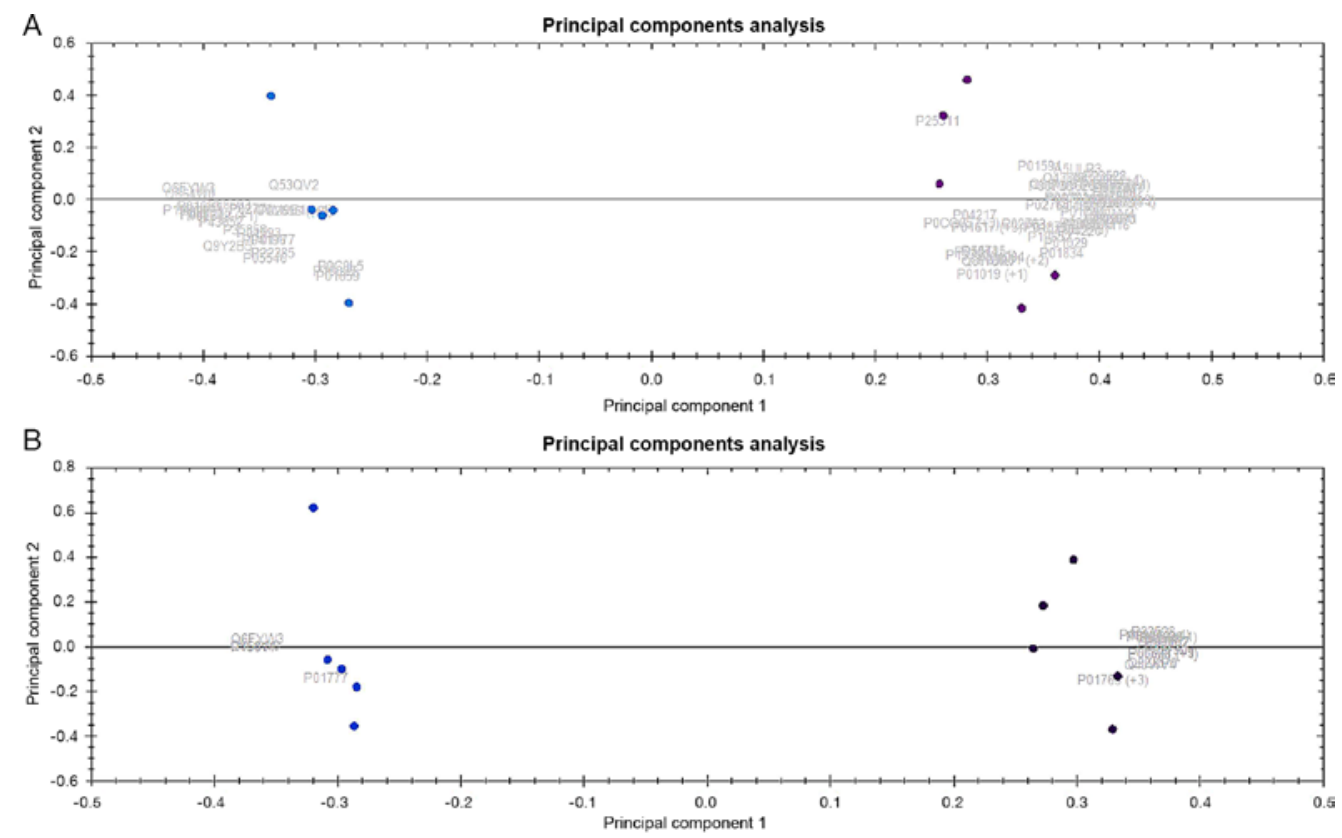

Figure 4. (A) Principal component analysis (PCA) plot of CML peripheral blood samples using the expression dataset of 164 identified proteins that were significantly differentially expressed $(>1.5$ - to $\infty$-fold change; $\mathrm{P}<0.05)$ between LT-MMR and P-No-MMR sample groups. The expression profiles of these proteins correctly predict patients with major molecular response (LT-MMR, blue) vs. no-major molecular response (P-No-MMR, purple) using principal component analysis. (B) Principal component analysis (PCA) plot of CML bone marrow samples using the expression dataset of 138 identified proteins that were significantly differentially expressed $(>1.5$ - to $\infty$-fold change; $\mathrm{P}<0.05)$ between LT-MMR and P-No-MMR sample groups. The expression profiles of these proteins correctly predict patients with long-term major molecular response (LT-MMR, blue) vs. persistent no-major molecular response (P-No-MMR, purple) using principal component analysis. The letters in grey color in the background represents the accession numbers of all the implicated proteins in the analysis. [Both images were generated using Progenesis QI for proteomics (Progenesis QIfp version 2.0.5387) (Nonlinear Dynamics/Waters)].

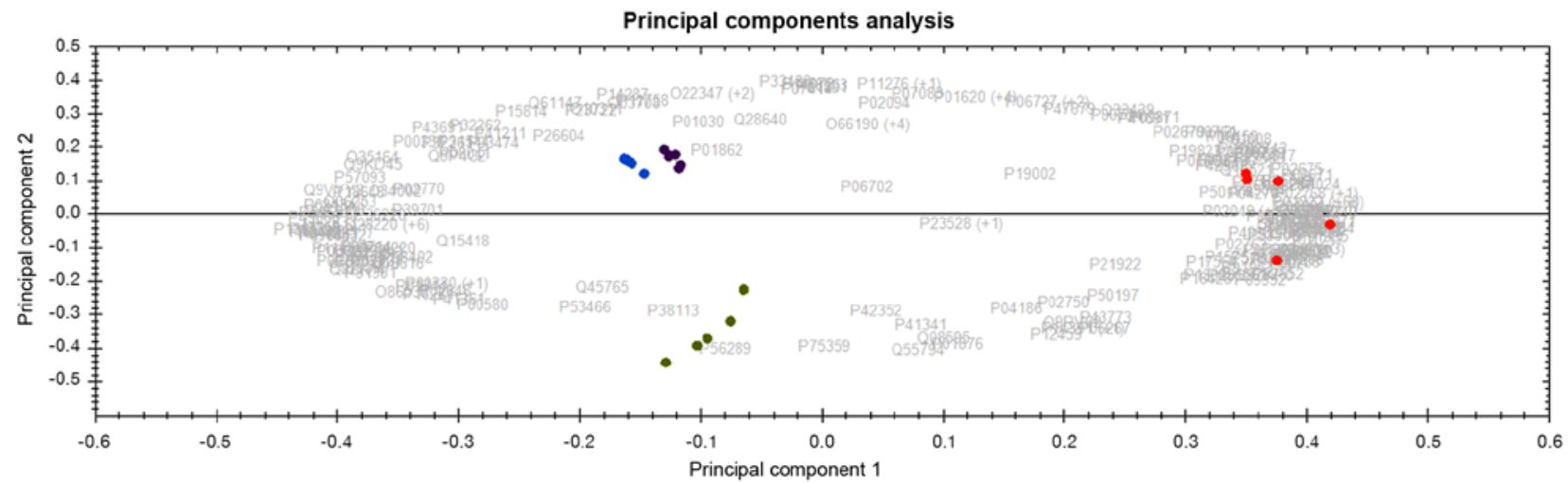

Figure 5. The same dataset from Fig. 4B (i.e. the expression of 138 identified bone marrow proteins that were significantly differentially expressed $(>1.5$ - to $\infty$-fold change; $\mathrm{P}<0.05$ ) between LT-MMR and P-No-MMR sample groups) separate all four sample groups including patients that stays on TKI after 1 year of imatinib Rx from patients ultimately requiring alternative treatment using principal component analysis. Long-term major molecular response (LT-MMR, blue), persistently no-major molecular response (P-No-MMR, purple, patients that stays on TKI after 1 year of imatinib Rx, green and patients ultimately requiring alternative treatment, red). The letters in grey color in the background represents the accession numbers of all the implicated proteins in the analysis. [The image was generated using Progenesis QI for proteomics (Progenesis QIfp version 2.0.5387) (Nonlinear Dynamics/Waters)]. Some of the identified proteins were implicated in hematological diseases as potential biomarkers using ingenuity pathway analysis as detailed in Fig. 6.

patients using quantitative label-free LC/MS/MS expression proteome analysis.

Approximately 700 proteins representing 280 unique protein species were identified (due to different protein isoforms). Only 164 of the 280 proteins were significantly differentially expressed between LT-MMR and P-No-MMR sample groups $(>1.5$ - to $\infty$-fold change; $\mathrm{P}<0.05)$ and accurately predict patients with major molecular response (LT-MMR) vs. No-major molecular response (P-No-MMR) using unsupervised principal component analysis (Fig. 4A). The list of identified differentially expressed proteins in PBP is described in Table IIA.

Similar to peripheral blood samples, $>700$ proteins representing 250 unique protein species were identified when similar analysis was done on bone marrow pooled samples from 8 LT-MMR patients and 8 P-No-MMR patients. One hundred and thirty-eight of the total identified proteins were significantly differentially expressed between LT-MMR and P-No-MMR bone marrow sample groups $(>1.5$ - to $\infty$-fold change, $\mathrm{P}<0.05$; Table IIB). These proteins predict accurately 


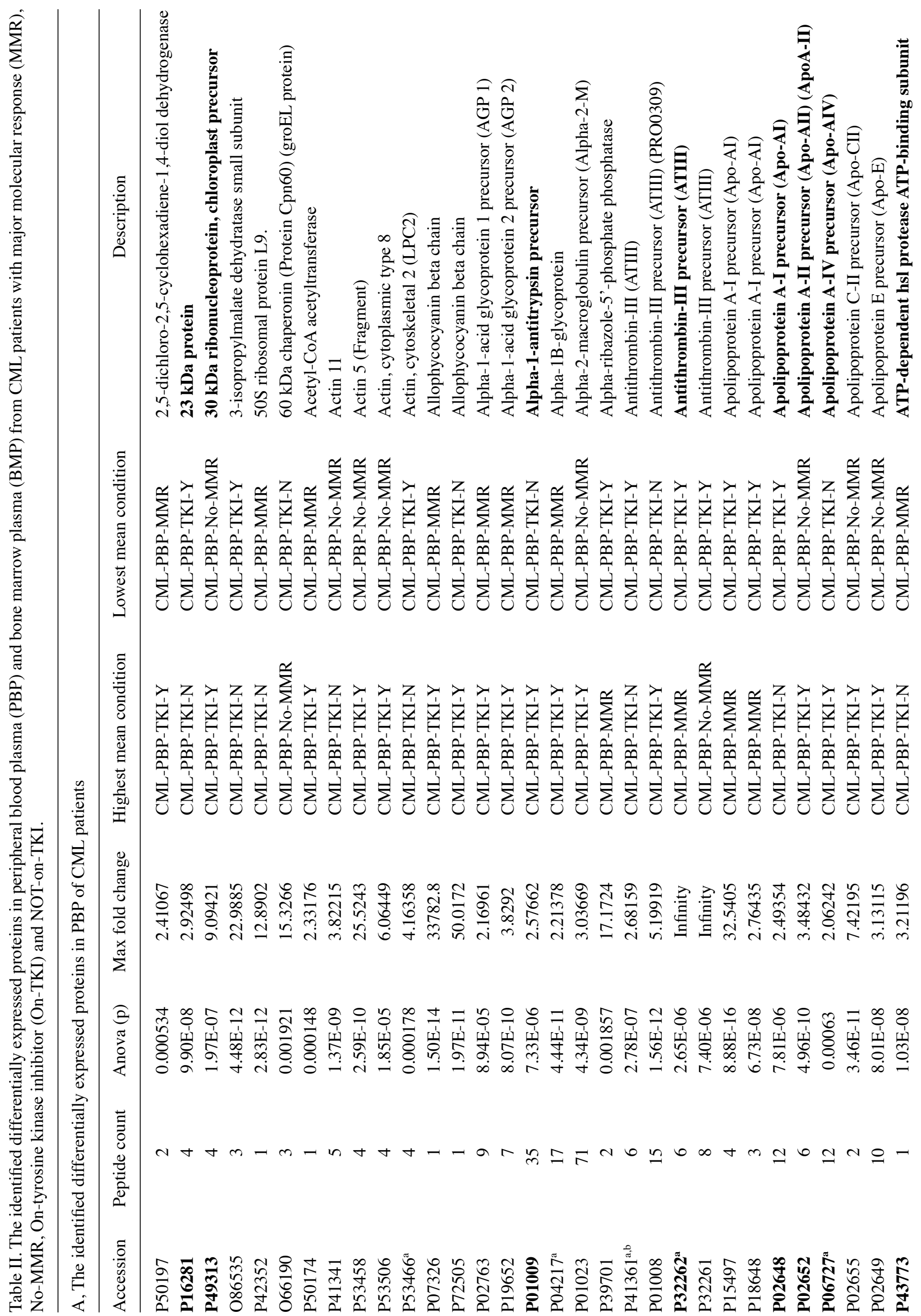




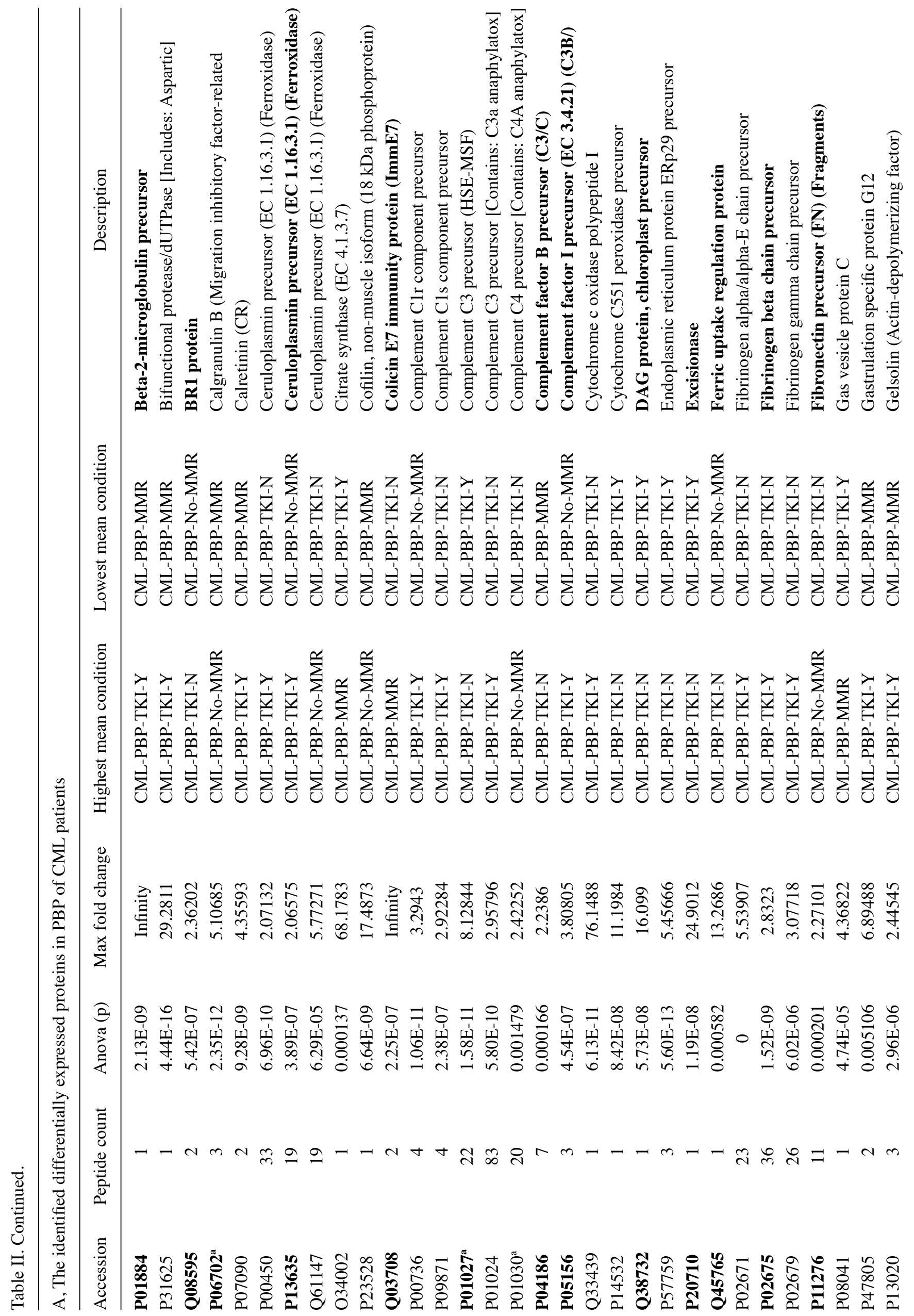




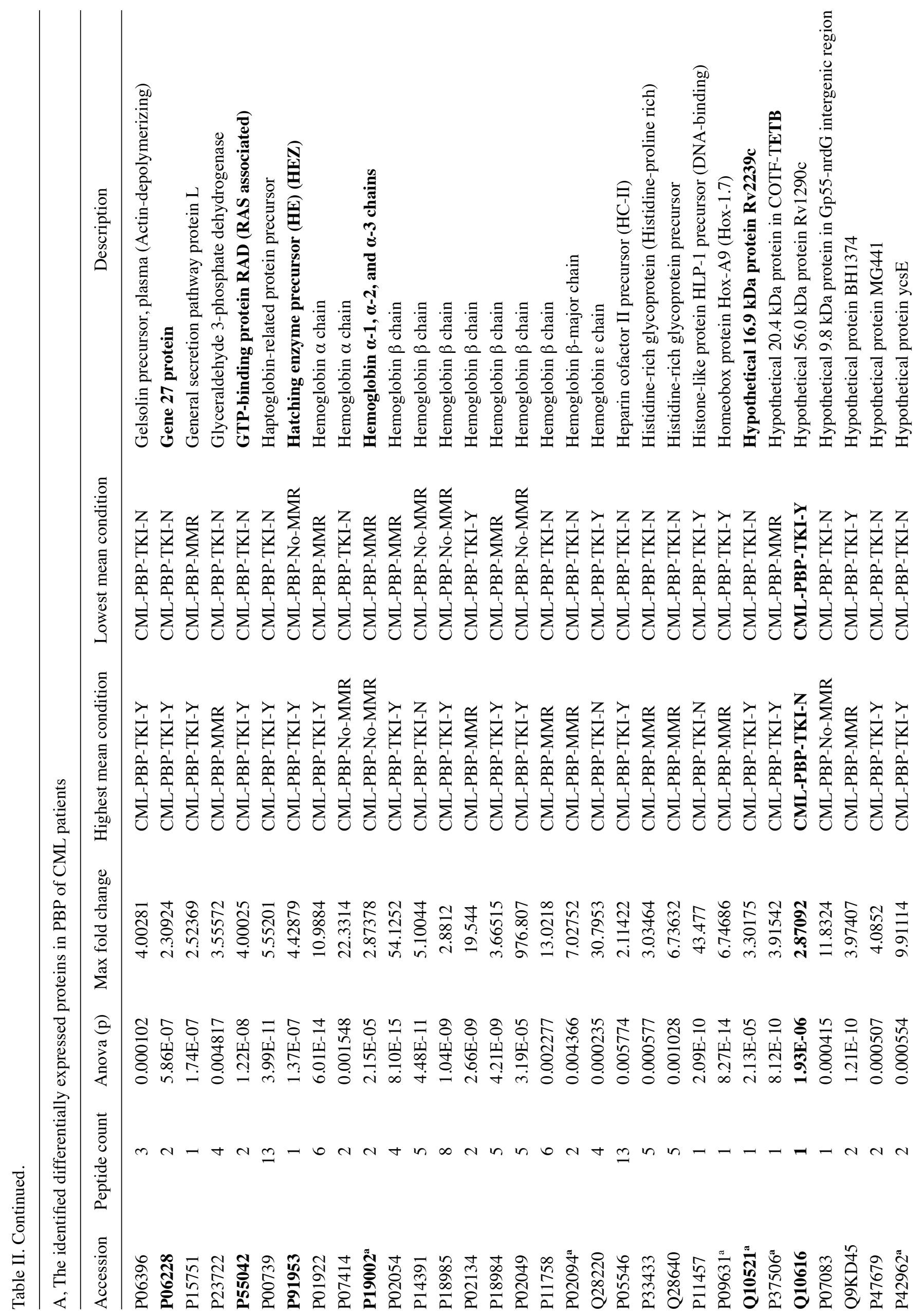




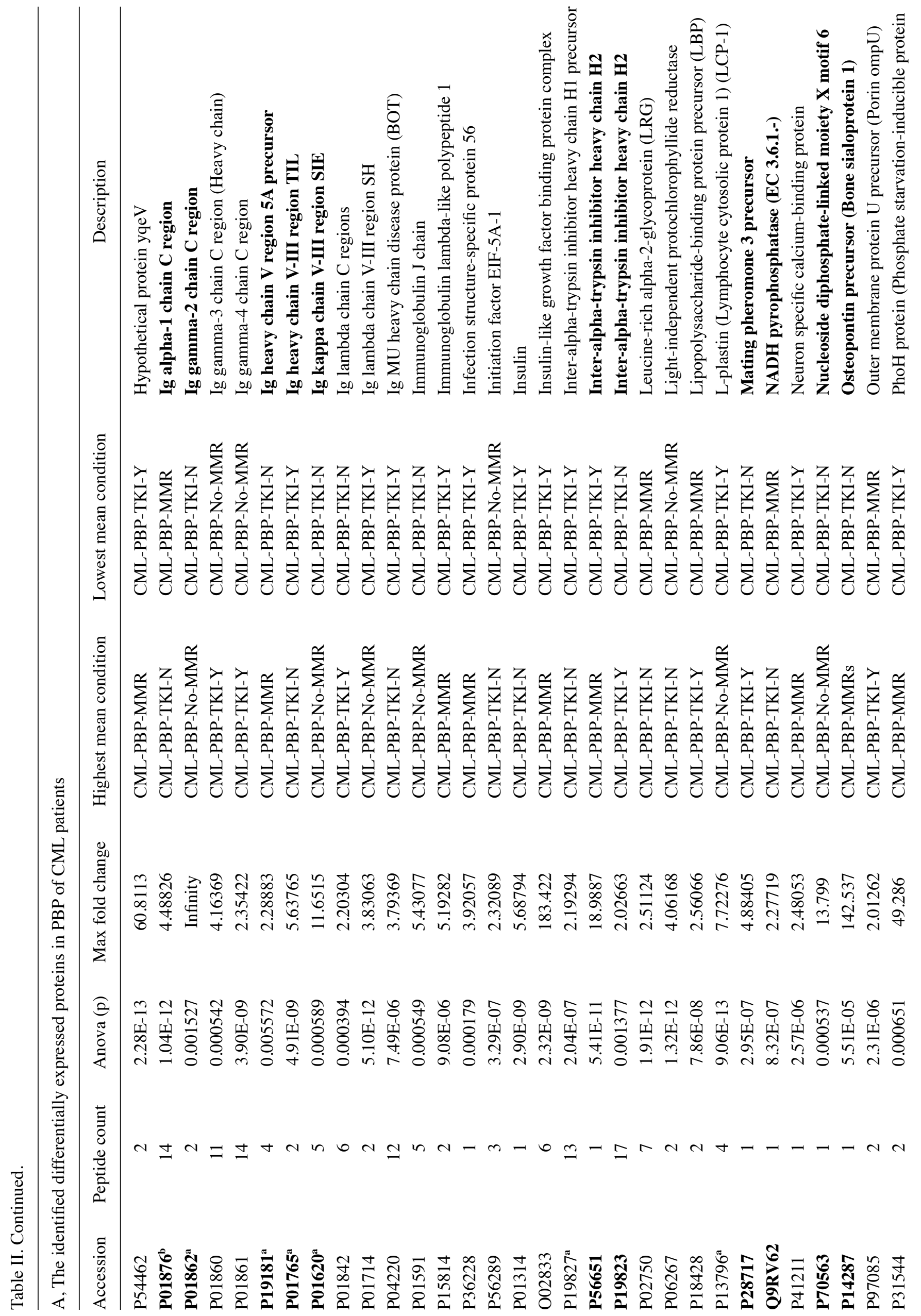




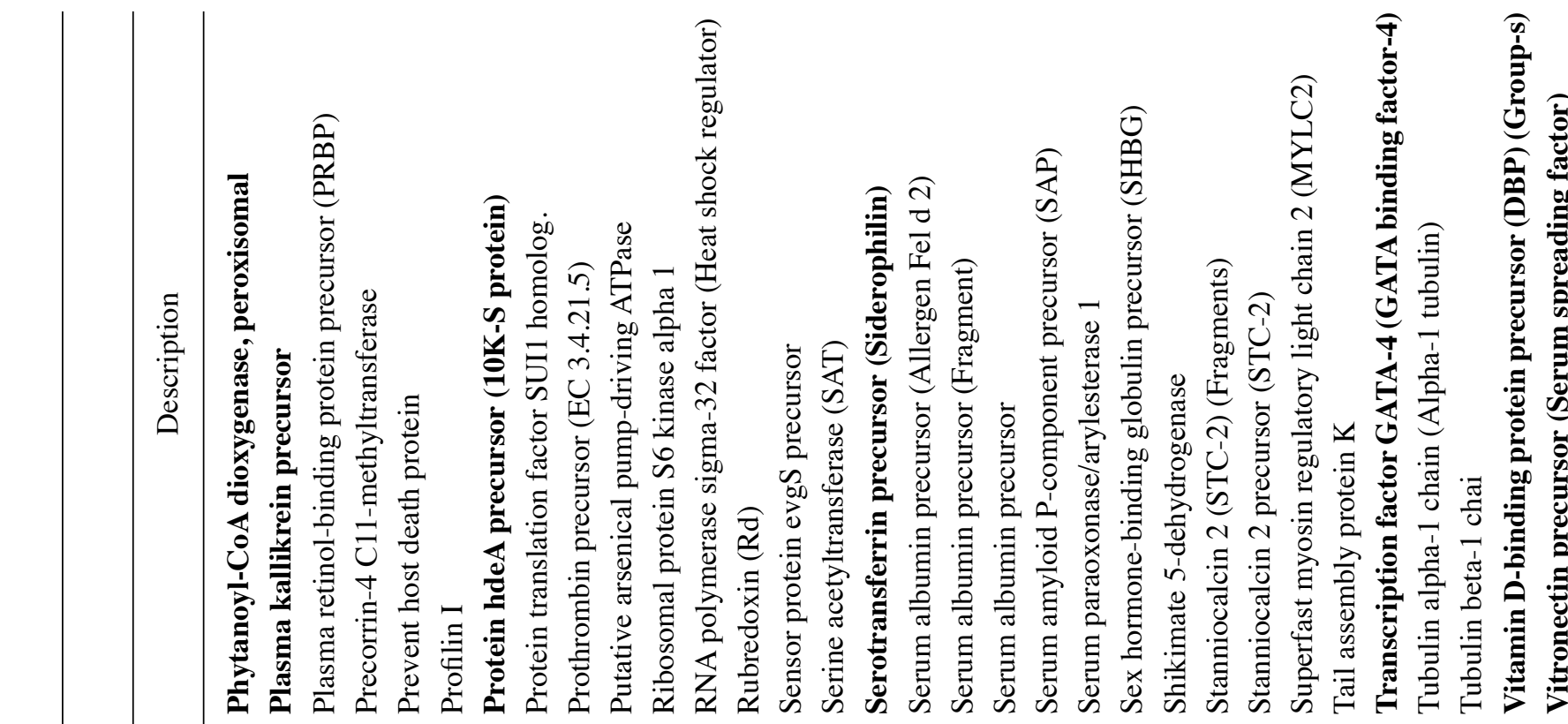

:

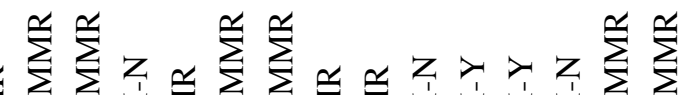

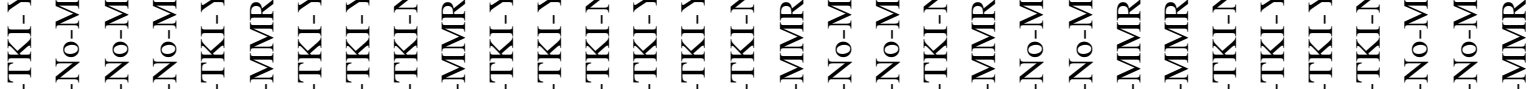

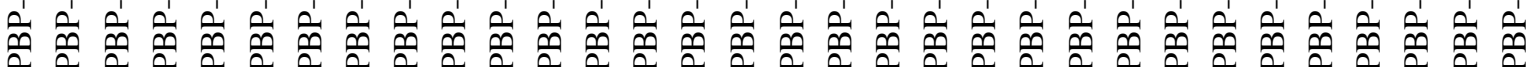

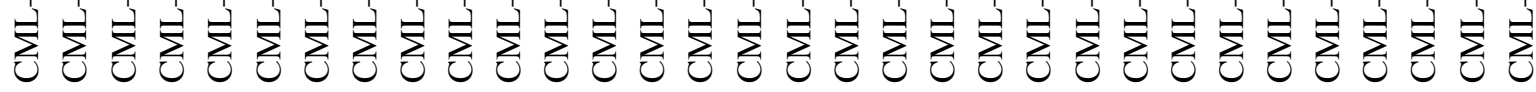

$\sum_{i}^{\infty} \lambda_{1}$

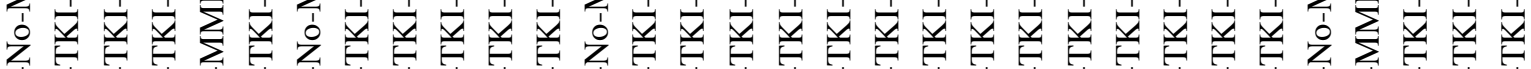

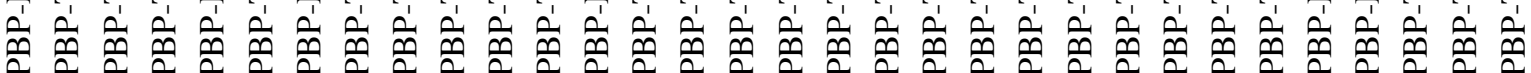

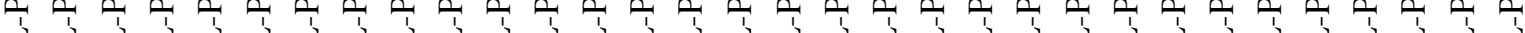

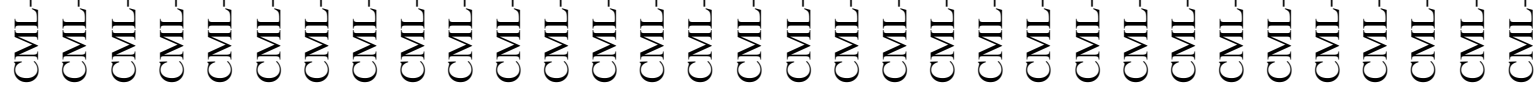

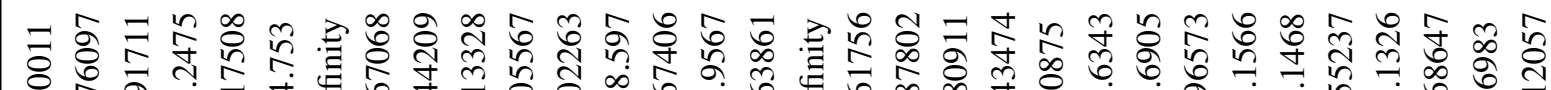

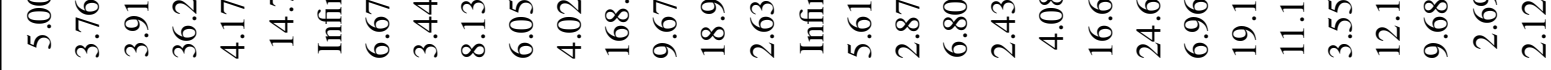

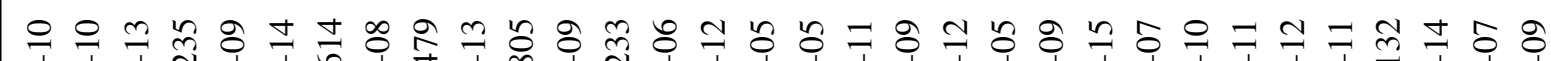
告

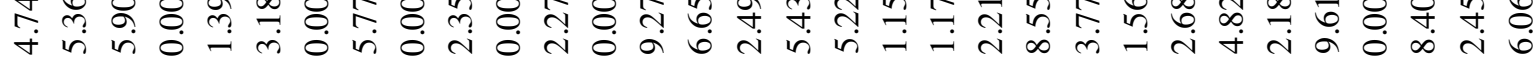

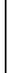

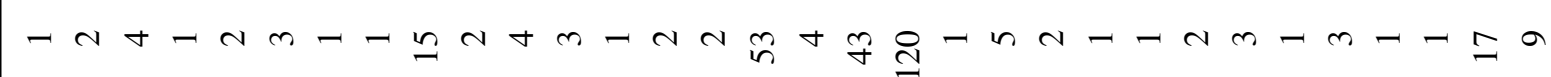

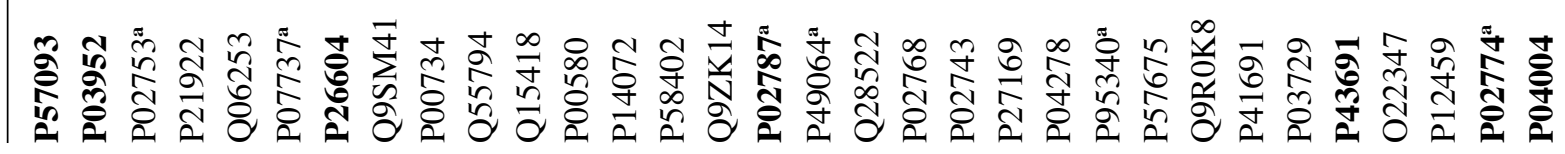




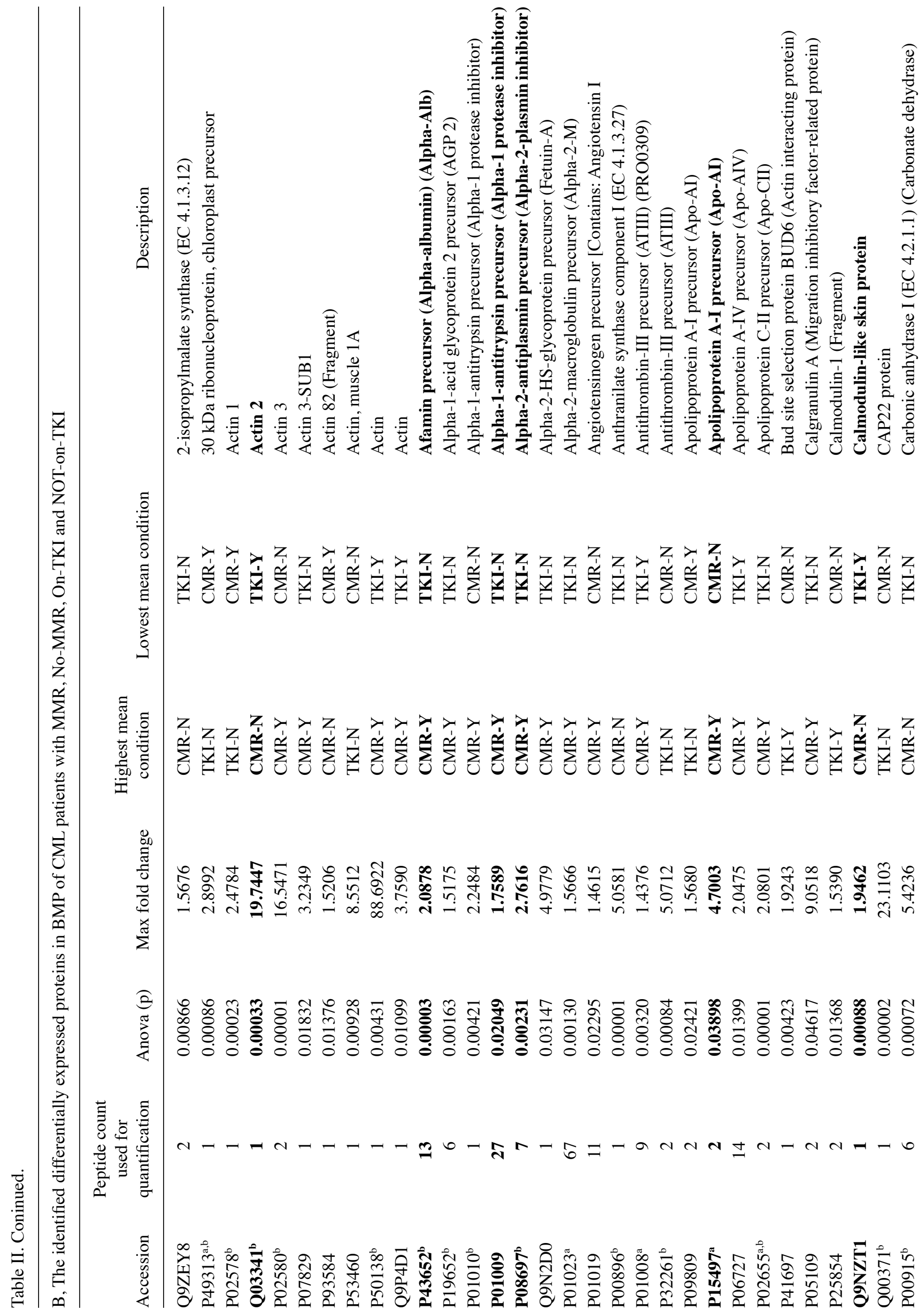




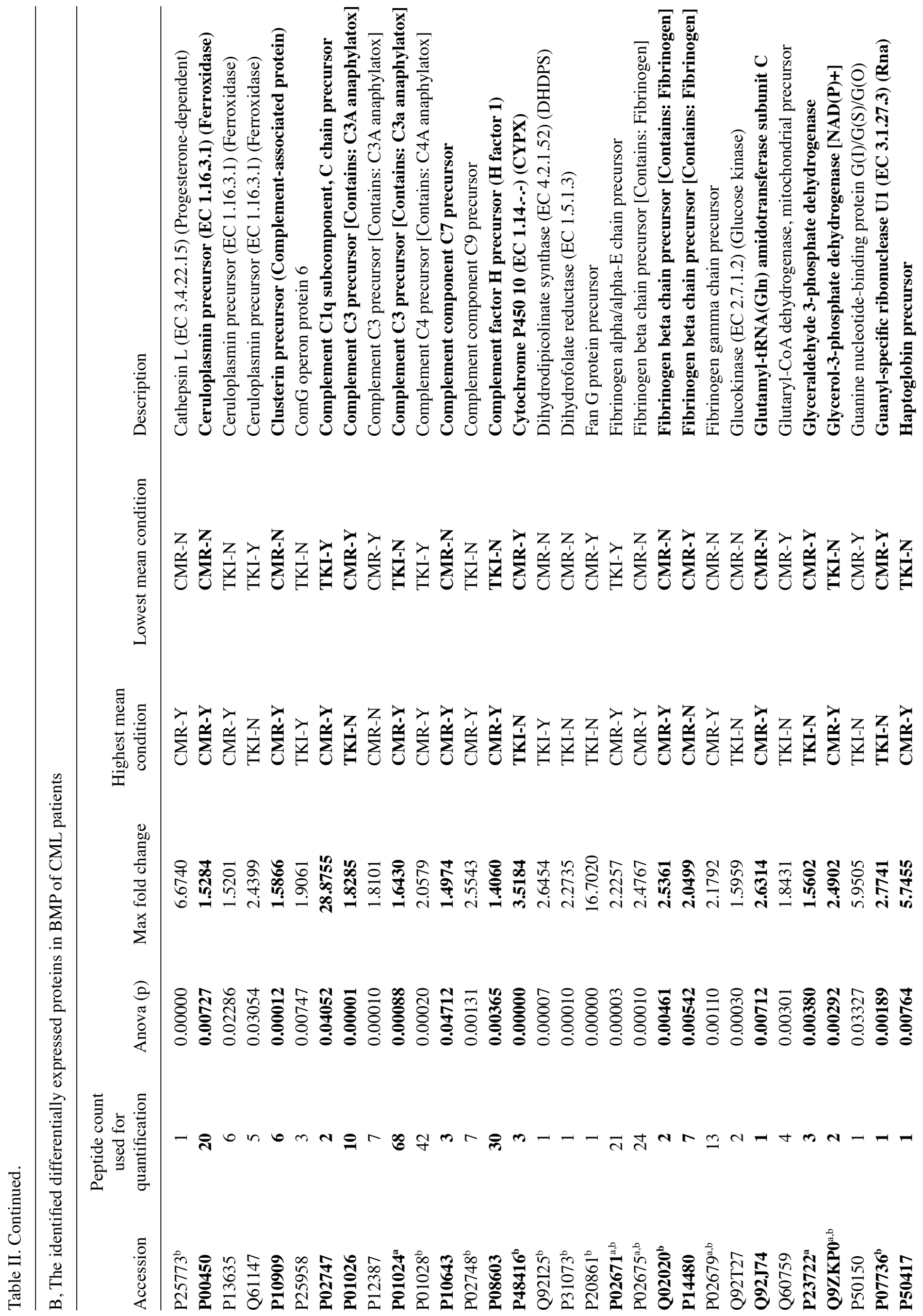




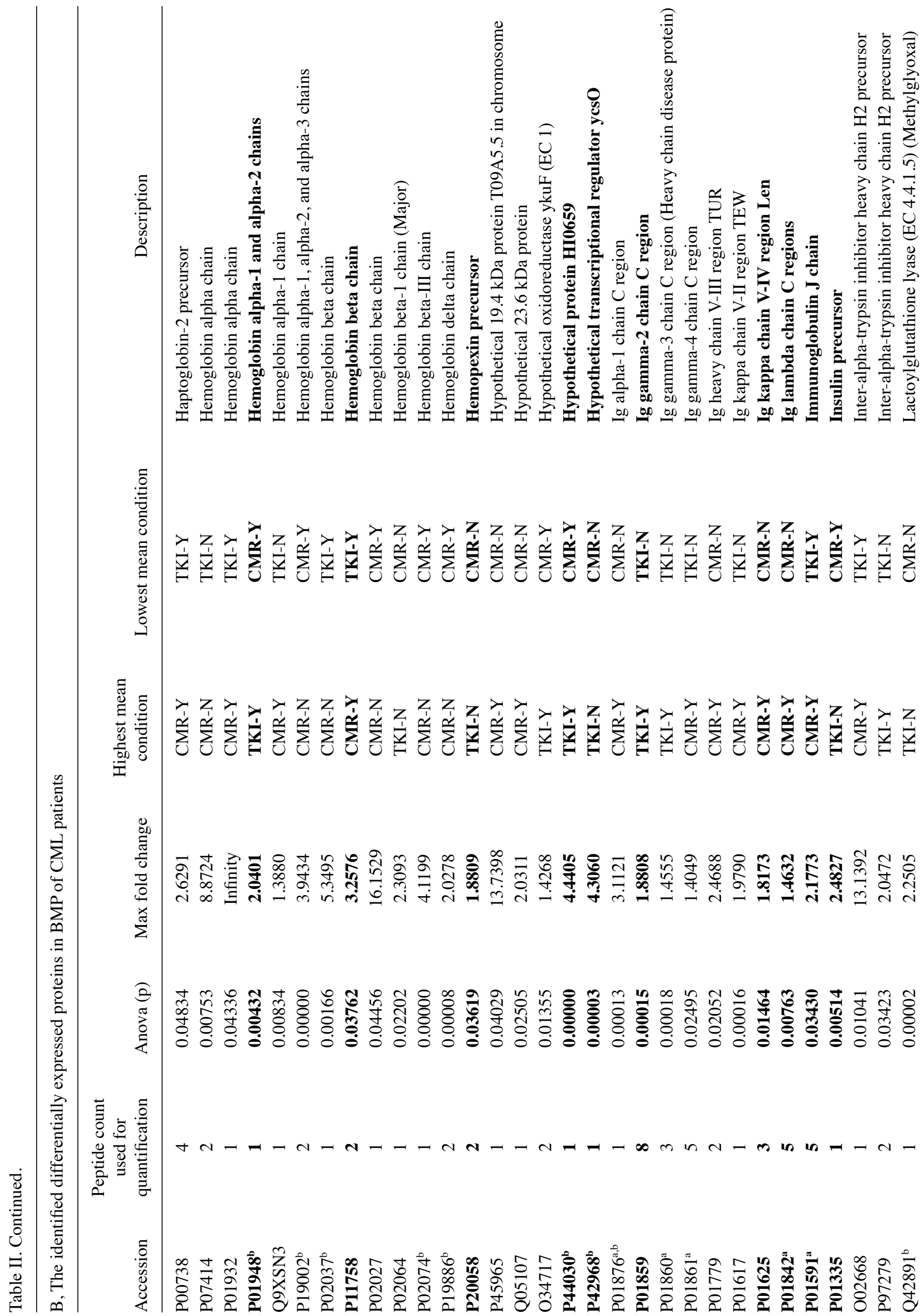




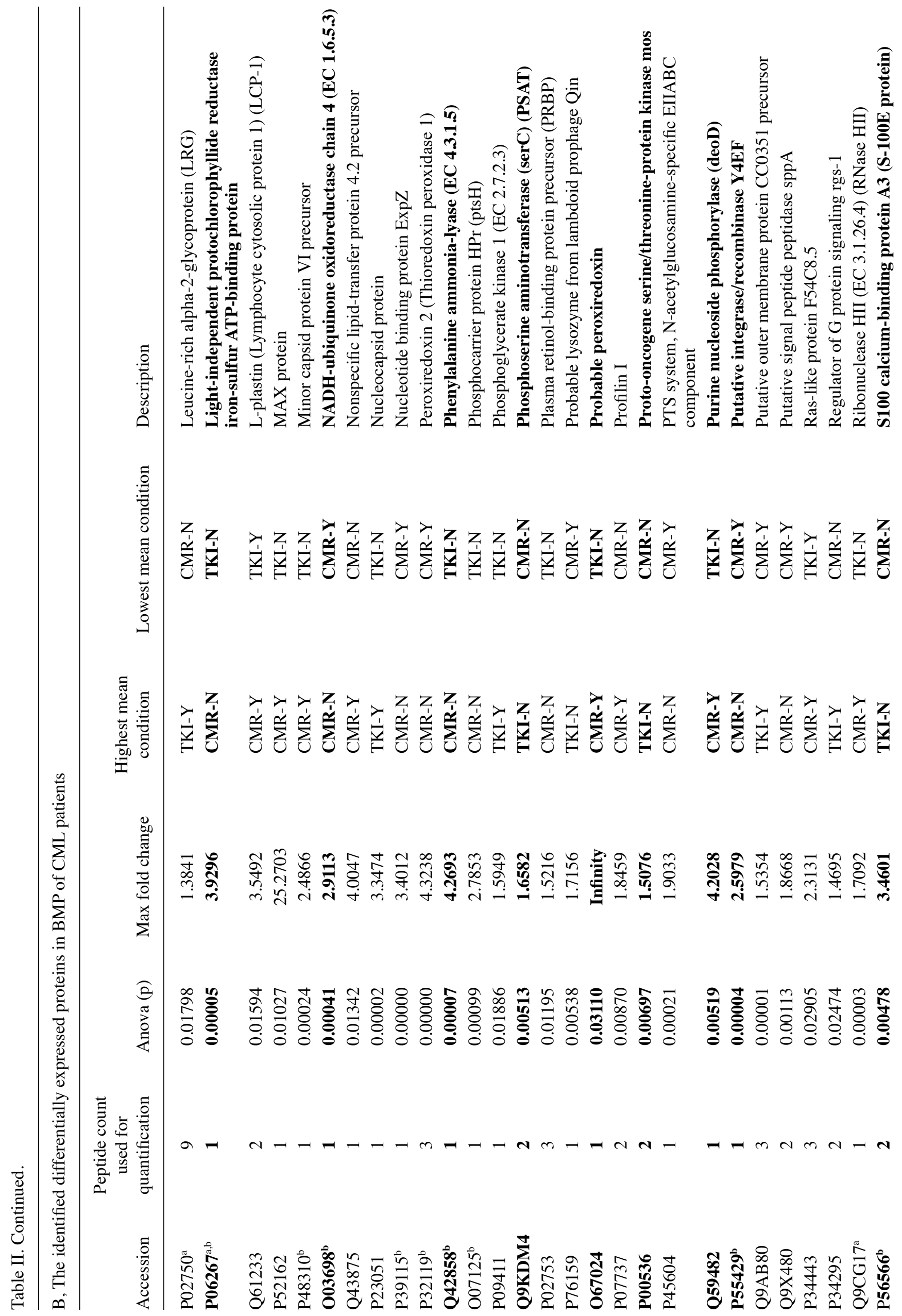




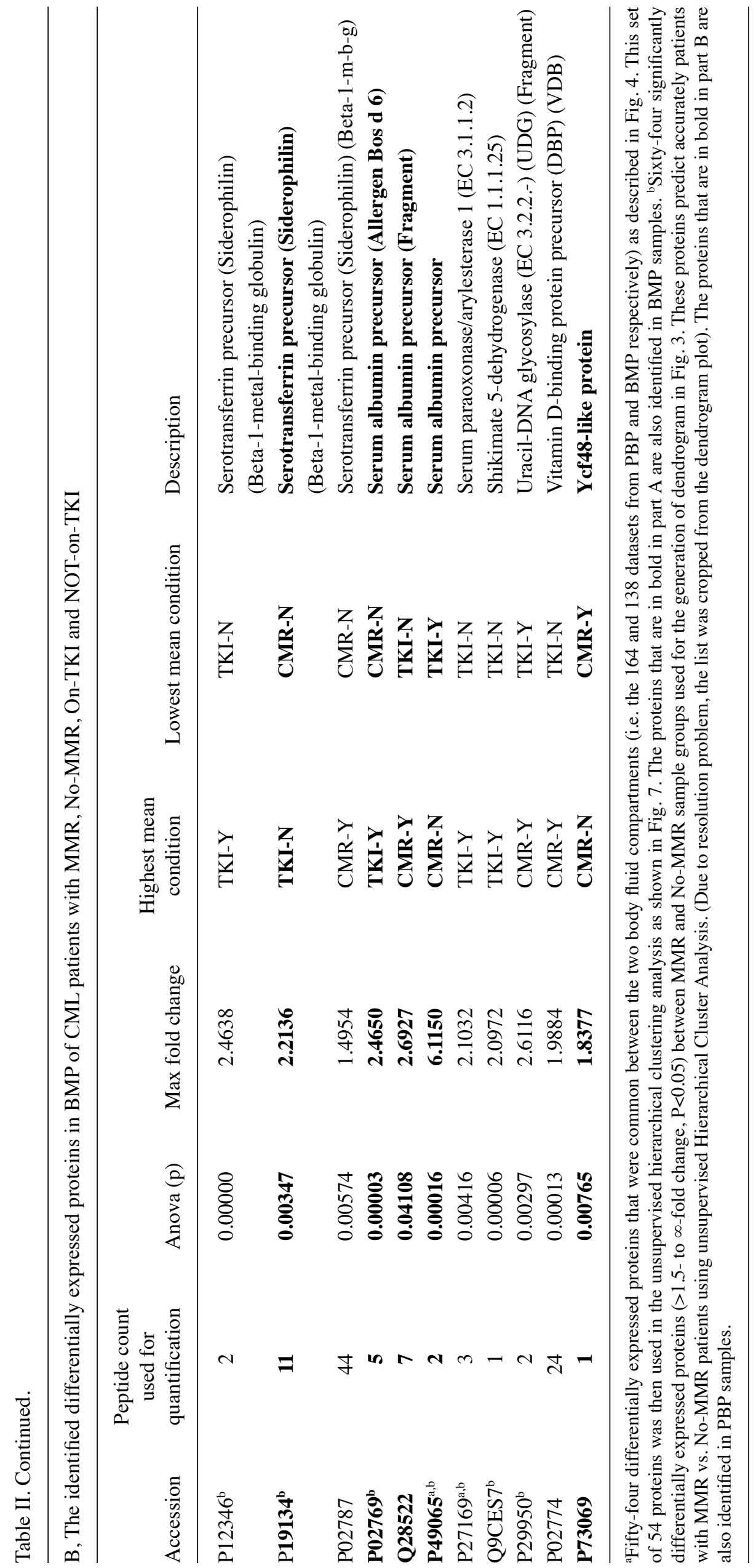



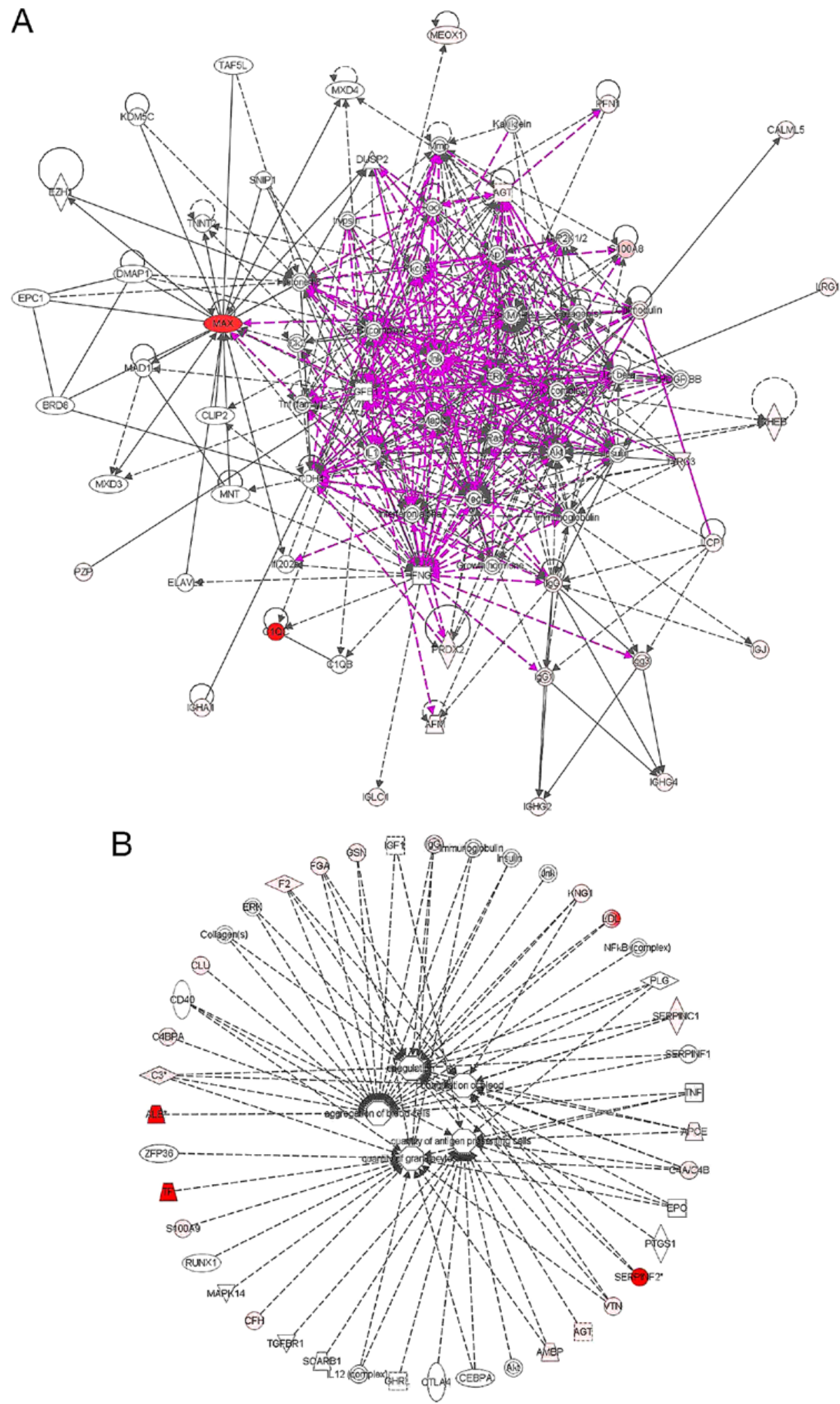

Figure 6. (A) Pathway analysis of network signaling of some of the identified proteins as represented in the ingenuity pathway analysis database. The analysis of the identified proteins is composed of 2 hematological disease related networks with over 100 associated molecules that were merged into one as shown above. The connections and the expression profiles of some of the identified proteins are as indicated. Red indicates an upregulated protein, and pink color is indicative of downregulation. A direct connection is by solid line and broken lines indicate an indirect interaction between different molecules. Other molecules outside the identified in this study are in grey color. (B) The functional characteristics and disease relatedness of some of the identified proteins were mapped in Ingenuity database. The majority of these molecules are located mostly in the plasma membrane, cytoplasm and extracellular space, while only a few are located in the nucleus. Some these molecules functions as enzymes, transporters, transcription regulator, or G-protein coupled receptor. Others act as kinases, peptidase or growth factor. Furthermore, some of these molecules as represented in multiple sub-signaling networks mostly regulate among others: Cell-To-Cell Signaling and Interaction, Hematological System Development and Function. Other implicated functional annotations include, aggregation of blood cells, coagulation, quantity of aggregate cells as well as quantity of granulocytes. [The network analysis was done and figure generated in ingenuity pathway analysis program (IPA v8.7)]. 


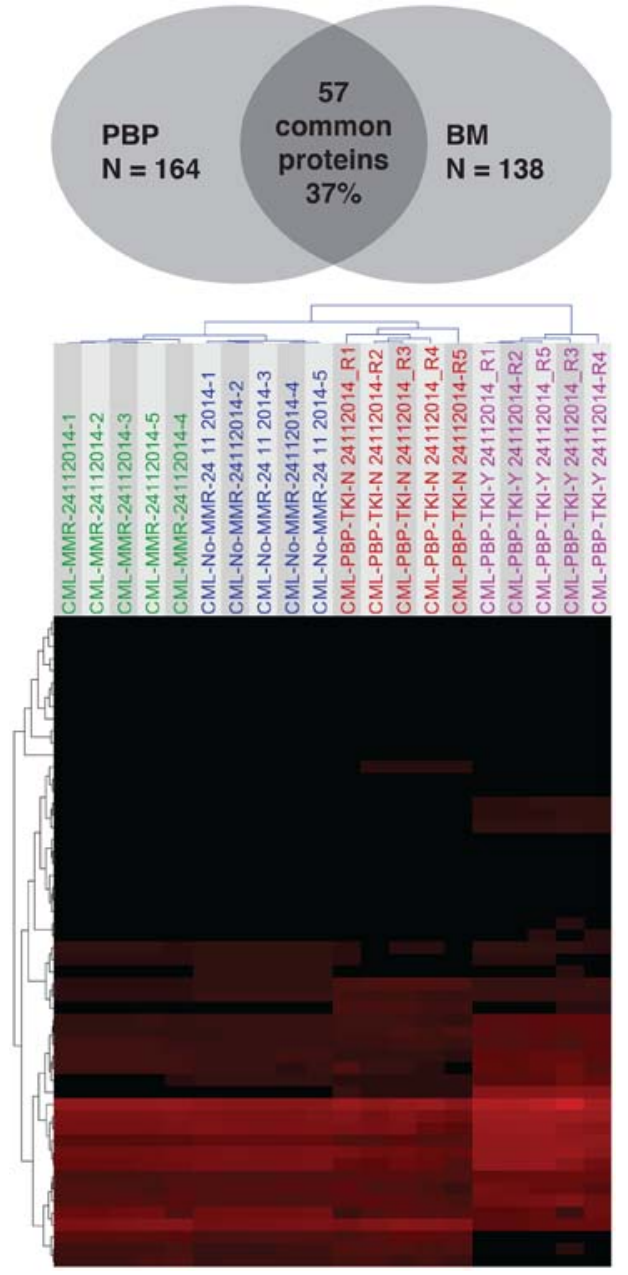

Figure 7. Unsupervised hierarchical cluster analysis of 54 identified differentially expressed proteins that are common in both bone marrow plasma (dataset of 138 proteins) and peripheral blood plasma (dataset of 164 proteins) of CML samples. The dendrogram shows correct prediction of patients with long-term major molecular response (LT-MMR, green), persistent no-major molecular response (P-No-MMR, blue), patients that stays on TKI after 1 year of imatinib Rx, purple and patients on alternative treatment outside TKI, red). The image was generated using J-Express Pro V 1.1 software program. (These 54 proteins used in generating this dendrogram plot are indicated with the letter a in Table II).

LT-MMR patients vs. P-No-MMR patients using unsupervised principal component analysis (Fig. 4B). These results were subsequently evaluated for comparisons with the patterns obtained in early treatment response at 6 months. Notably, the pattern and accuracy of clustering of samples is very similar to that observed with the hierarchical cluster analysis plots at 6 months (Fig. 3).

Protein fingerprinting for prediction of treatment options for individualized therapy. Towards achieving the goal of personalized medicine, the above observed differentially expressed proteins between samples derived from LT-MMR patients vs. P-No-MMR patients were evaluated for their potential for objective prediction of treatment options for some of these cohorts of CML patients. Interestingly, the panel of 164 and 138 differentially expressed protein datasets derived from peripheral blood plasma (PBP) and bone marrow (BM) respectively, also discriminates patients that stay on IM after
1 year of treatment from patients that ultimately required alternative treatment options (second generation TKI/others) (Fig. 5). Following $>2$ years of follow-up of these patients the same dataset of potential protein biomarkers could still accurately separate all analyzed sample groups into their respective molecular response and treatment sub groups, indicating their usefulness for treatment monitoring as well as prediction of best choice of therapy for individual patient. Some of the identified proteins were implicated in hematological diseases as potential biomarkers using ingenuity pathway analysis (IPA) (Fig. 6). Functional annotations/ disease affiliations of some of these proteins implicated in CML are further described under discussion below.

Identification of protein changes in BM as a reflection of detectable changes in peripheral blood. One of the main goals of this study was to identify/develop disease-specific/ disease-associated protein biomarkers seen in bone marrow tissue as well as in peripheral blood plasma. This would subsequently allow monitoring of such biomarker proteins in peripheral blood, rather than bone marrow, demanding less invasive procedures for objective prediction of individual's best treatment options and prognostic monitoring of CML patients. We therefore explored the possibility whether the proteins that are significantly differentially expressed in bone marrow do also show similar expression pattern in peripheral blood. With this in mind, we calculated how many of the 164 differentially expressed proteins in peripheral blood and the 138 protein dataset in bone marrow are common to both body compartments. We found that only 54 proteins $(\sim 35 \%)$ were in common between the two 164 and 138 datasets as described above. This set of 54 proteins was then subjected to unsupervised hierarchical clustering and correspondence analyses. As shown in Fig. 7, all sample groups were distinctively separated into four response subtypes using unsupervised hierarchical cluster analysis. The common proteins between the two body fluid compartments were highlighted in bold in Table II.

Validation by western blot analysis of some of the identified proteins. In an attempt to validate some of the differentially expressed proteins, we have used immunoblotting analysis. Nine individual samples consisting of 4 samples not included in the proteomics analysis and 5 other samples from the proteomics analyzed sample groups were tested for their expression of haptoglobin and hemoglobin using specific antibodies against these proteins. The expression levels of these proteins across all sample groups were consistent with the average protein normalized levels seen with label-free quantitative LC/MS/MS analysis (Fig. 8). Large scale validation of the majority of these proteins was beyond the scope of this study in order to develop limited panel of markers for clinical trial in a later study.

\section{Discussion}

Clinical and molecular diagnosis of most hematological malignancies including CML can be accurately made; however, prediction of treatment response elude the currently available tools for patient care. 


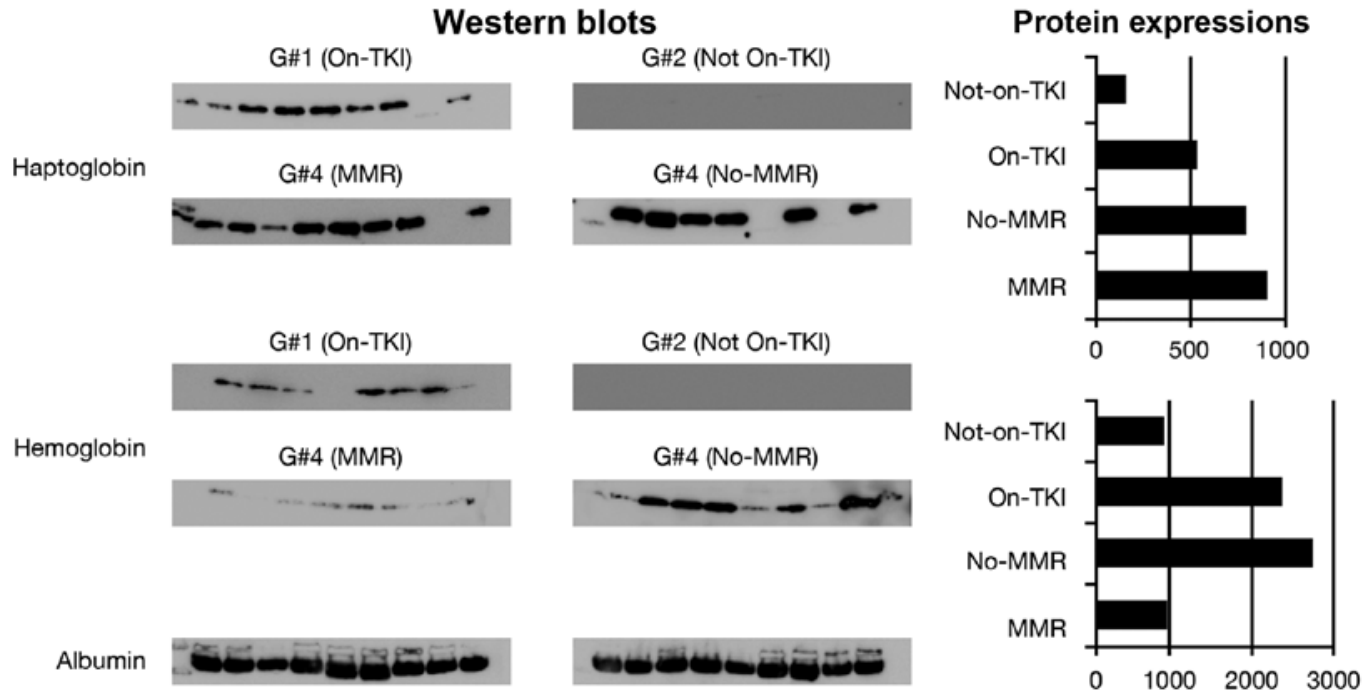

Figure 8. Western blots validation analysis abundance of 2 of the identified differentially expressed proteins. Each lane indicates the expression of 9 individual samples in each of the four sample groups representing long-term major molecular response to imatinib (LT-MMR), persistently no major molecular response (P-No-MMR), patients that stay on TKI after 1 year of imatinib treatment (On-TKI) and patients that ultimately required alternative treatment options, i.e. second generation TKI/others (Not-On-TKI). Albumin was used as internal standard for normalization. The histogram bars are the corresponding average group protein expressions of the two protein haptoglobin and hemoglobin using label-free LC/MS/MS expression analysis platform.

A subset of significantly differentially expressed proteins from both peripheral blood and bone marrow were selected for their ability to discriminate samples derived from CML patients that responded differently to initial first line treatment with imatinib. Our strategy of proteomics mining of BM and PBP from the same individual patient would provide unique possibility to identify biomarkers from both sources thus, entailing less invasive procedures.

Report of microarray analysis of peripheral blood and bone marrow of CML samples in blast crisis cells, has been shown with demonstrable biological changes between two bodily fluids (19). Our analysis of peripheral blood samples of 164 differentially expressed proteins show that all samples were correctly classified and similar result was observed with 138 protein changes in bone marrow samples as shown in Fig. 4. Only 54 proteins were shown to be commonly differentially expressed between blood dataset and bone marrow protein dataset in the present study, supporting our notion that it might be possible to identify significant changes in the bone marrow of CML patients that are measurable at peripheral blood compartment for routine diagnostics.

We have attempted to use both the BMP and PBP datasets that accurately predict patients MMR status for possible prediction of patients that continue to stay on IM after 1 year of treatment vs. those that ultimately required alternative treatment options (second generation TKI/others). Thus, the expression of the 158 protein changes in BM between MMR and No-MMR were further evaluated in 16 unrelated patients that stay on TKI after 1 year of imatinib treatment from patients that ultimately required alternative treatment options (second generation TKI/others). We found four distinct clusters with samples with MMR and No-MMR being very closely separated (not as distinct as in Fig. 4), while patients that stay on TKI (i.e. after 1 year of imatinib) treatment were distantly separated from patients that ultimately required alternative treatment options (second generation TKI/others) as shown in Fig. 5, meaning that it will be challenging to construct a universal model for management of CML patients and that prognostic datasets need to be created for each specific response type.

We have used two independent proteomics analysis platforms in the present study. The expression profiles of 2-DE protein spots successfully discriminated two sample groups of CML patients with MMR and No-MMR. We recognized the inherent limitation of 2-DE based studies (20-22) hence, we have in addition used label-free quantitative protein expression using high definition liquid chromatography tandem mass spectrometry (LC/MS/MS) to extensively map the proteome of bone marrow as well as peripheral blood samples.

Previous studies have used multivariate statistical algorithms and artificial learning models to predict cancer prognosis and for grading different solid tumors (15,23-28). The majority of these studies reported varying degrees of sensitivity and specificity based on evaluation of different clinical parameters $(20,24)$.

Gene expression studies on hematological disease have been largely carried out by analysis of DNA or RNA microarrays. These genomics studies have indicated the potentials of large scale analysis of gene expression towards better understanding the molecular basis of leukemogenesis and that this information could potentially be useful in the classification of subtypes of hematological malignancies $(19,29,30)$. In a recent study of CLL samples, Alsagaby and colleagues used combined transcriptomics and proteomics analyses to unravel the heterogeneity of gene expression patterns as well attempting to identify proteins that are implicated in prognosis of chronic lymphocytic leukemia (31). Recent studies have attempted to evaluate protein changes between imatinib sensitive and resistance samples (32) as well as to better understand the molecular mechanism in therapy resistance at the level of bone marrow extracellular fluid in CML (33). 
Our initial analysis of 64 differentially expressed proteins of peripheral blood for prognostic monitoring of early CML treatment response at 6 months was encouraging and led us into extensive analysis of samples with sustained long-term MMR against patients that persistently could not achieve MMR.

Some of the identified proteins in the bone marrow of the 138 dataset for the prolonged and sustained MMR vs. persistent No-MMR were further evaluated for their functional characteristics and their hematological disease relevance using ingenuity pathway analysis (IPA). In the canonical pathway analysis of network signaling of identified proteins, only 48 of the 138 identified differentially expressed proteins were represented in the IPA database. The analysis of the identified proteins is composed of multiple networks of which, one is implicated in hematological disorders. The cellular localization, interconnections and functional annotation as well as the expression profile of some of these 48 identified molecules are as detailed in Fig. 6A. A review of some of these molecules showed that they mostly regulate among others: cell-to-cell signaling and interaction, hematological system development and function, aggregation of blood cells, coagulation, as well as quantity of granulocytes as indicated in Fig. 6. Among the identified proteins in this study is TYRO3 protein tyrosine kinase, a member of TAM family of receptor tyrosine kinases (RTKs) and known for their role as regulator of cellular proliferation, migration and survival processes, as well as maintenance of blood coagulation equilibrium (34). We observed connection of TYRO 3 in AKT/P13K pathway; similar to that previously described (34-36).

The S100A8 is a calcium-binding protein of the S100 family and have been described to be associated with myeloid differentiation (37). We observed a more than 9-fold differential expression of S100A8 and in the network connecting with RAS, TGFb, MAPK and MMP. The S-100 protein has been previously reported as a useful marker in juvenile chronic myeloid leukemia (JCML) as well as myeloid leukemia cutis (LC) $(38,39)$.

Overexpression of MYC has been associated with CML with poor response to imatinib $(40,41)$. We observed a more than 25-fold differential expression of MYC associated factor $\mathrm{x}$ in this study.

Altogether our findings indicate that rather than the use of a single marker, analyses of a panel of protein markers have the potential to provide better insight into complex biologic processes towards better prognostication of CML patients.

We recognize the limitation of this study as samples were prospectively collected and patients observed over the years for their treatment responses. One other issue with this study is the low number of patients enrolled in different clinical and molecular response groups; hence we have limited the analysis to evaluation of patients based on MMR and whether or not they are on IM or alternative treatment option (second generation TKI/others).

In conclusion, we have identified protein signatures capable of prediction of molecular response and choice of therapy for CML patients at 6 months and beyond using expression proteomics as objective stratification of CML patients for treatment options.

Although these results are very promising, we recognized that analysis of much larger materials of patients with similar treatments and responses will be necessary to validate if clustering analysis can be used as a routine prognostic tool for CML patients.

These proteins might be valuable once validated, to complement the currently existing parameters for reliable and objective prediction of disease progression, monitoring treatment response and clinical outcome of CML patients as a model of personalized medicine.

\section{Acknowledgements}

We thank Dr Abdelilah Aboussekhra for critical review, as well as Mr. Melvin Velasco, Mr. Parvez Siddiqui, Mr. Romeo Caracas and Ms.Tusneem Elhassan for technical assistant. We acknowledge the assistant and support of Mr. Faisal Al Otaibi and the logistics and purchasing department, RC, KFSH\&RC. The present study was supported by the Research Center Administration, KFSH\&RC, Riyadh, Saudi Arabia (RAC\# 2050 040).

\section{References}

1. Bartram CR, de Klein A, Hagemeijer A, van Agthoven T, Geurts van Kessel A, Bootsma D, Grosveld G, Ferguson-Smith MA Davies T, Stone M, et al: Translocation of c-abl oncogene correlates with the presence of a Philadelphia chromosome in chronic myelocytic leukaemia. Nature 306: 277-280, 1983.

2. Faderl S, Talpaz M, Estrov Z, O'Brien S, Kurzrock R and Kantarjian HM: The biology of chronic myeloid leukemia. $N$ Engl J Med 341: 164-172, 1999.

3. Goldman JM and Melo JV: Chronic myeloid leukemia--advances in biology and new approaches to treatment. N Engl J Med 349: 1451-1464, 2003.

4. Kantarjian HM, Kurzrock R and Talpaz M: Philadelphia chromosome-negative chronic myelogenous leukemia and chronic myelomonocytic leukemia. Hematol Oncol Clin North Am 4: 389-404, 1990.

5. Martiat P, Michaux JL and Rodhain J: Philadelphia-negative (Ph-) chronic myeloid leukemia (CML): Comparison with $\mathrm{Ph}+\mathrm{CML}$ and chronic myelomonocytic leukemia. The Groupe Français de Cytogénétique Hématologique. Blood 78: 205-211, 1991.

6. Santucci MA, Saglio G and Tura S: Pathogenesis and progression of chronic myeloid leukemia. Haematologica 81: 63-76, 1996.

7. Goldman JM: Chronic myeloid leukemia: A historical perspective. Semin Hematol 47: 302-311, 2010.

8. Kantarjian HM, O'Brien S, Smith TL, Rios MB, Cortes J, Beran M, Koller C, Giles FJ, Andreeff M, Kornblau S, et al: Treatment of Philadelphia chromosome-positive early chronic phase chronic myelogenous leukemia with daily doses of interferon alpha and low-dose cytarabine. J Clin Oncol 17: 284-292, 1999.

9. Druker BJ, Sawyers CL, Kantarjian H, Resta DJ, Reese SF, Ford JM, Capdeville R and Talpaz M: Activity of a specific inhibitor of the BCR-ABL tyrosine kinase in the blast crisis of chronic myeloid leukemia and acute lymphoblastic leukemia with the Philadelphia chromosome. N Engl J Med 344: 1038-1042, 2001.

10. Gambacorti-Passerini C, le Coutre P, Mologni L, Fanelli M, Bertazzoli C, Marchesi E, Di Nicola M, Biondi A, Corneo GM, Belotti D, et al: Inhibition of the ABL kinase activity blocks the proliferation of $\mathrm{BCR} / \mathrm{ABL}^{+}$leukemic cells and induces apoptosis. Blood Cells Mol Dis 23: 380-394, 1997.

11. Kantarjian HM, Cortes JE, O'Brien S, Giles F, Garcia-Manero G, Faderl S, Thomas D, Jeha S, Rios MB, Letvak L, et al: Imatinib mesylate therapy in newly diagnosed patients with Philadelphia chromosome-positive chronic myelogenous leukemia: High incidence of early complete and major cytogenetic responses. Blood 101: 97-100, 2003.

12. Kantarjian H, Talpaz M, O'Brien S, Garcia-Manero G, Verstovsek S, Giles F, Rios MB, Shan J, Letvak L, Thomas D, et al: High-dose imatinib mesylate therapy in newly diagnosed Philadelphia chromosome-positive chronic phase chronic myeloid leukemia. Blood 103: 2873-2878, 2004. 
13. Hughes TP and Branford S: Monitoring disease response to tyrosine kinase inhibitor therapy in CML. Hematology Am Soc Hematol Educ Program 2009: 477-487, 2009.

14. Alaiya AA1, Franzén B, Hagman A, Dysvik B, Roblick UJ, Becker S, Moberger B, Auer G and Linder S: Molecular classification of borderline ovarian tumors using hierarchical cluster analysis of protein expression profiles. Int J Cancer 98: 895-899, 2002.

15. Alaiya AA, Franzén B, Hagman A, Silfverswärd C, Moberger B, Linder S and Auer G: Classification of human ovarian tumors using multivariate data analysis of polypeptide expression patterns. Int J Cancer 86: 731-736, 2000.

16. Alaiya A, Fox J, Bobis S, Matic G, Shinwari Z, Barhoush E, Márquez M, Nilsson S and Holmberg AR: Proteomic analysis of soft tissue tumor implants treated with a novel polybisphosphonate. Cancer Genomics Proteomics 11: 39-49, 2014.

17. Al-Moghrabi N, Nofel A, Al-Yousef N, Madkhali S, Bin AmerSM Alaiya A, Shinwari Z, Al-Tweigeri T, Karakas B, Tulbah A, et al: The molecular significance of methylated BRCA1 promoter in white blood cells of cancer-free females. BMC Cancer 14: 830, 2014.

18. Li GZ, Vissers JP, Silva JC, Golick D, Gorenstein MV and Geromanos SJ: Database searching and accounting of multiplexed precursor and product ion spectra from the data independent analysis of simple and complex peptide mixtures. Proteomics 9 : 1696-1719, 2009.

19. Nowicki MO, Pawlowski P, Fischer T, Hess G, Pawlowski T and Skorski T: Chronic myelogenous leukemia molecular signature. Oncogene 22: 3952-3963, 2003.

20. Alaiya A, Al-Mohanna M and Linder S: Clinical cancer proteomics: Promises and pitfalls. J Proteome Res 4: 1213-1222, 2005.

21. Celis JE: Gel-based proteomics: What does MCP expect? Mol Cell Proteomics 3: 949, 2004.

22. Celis JE and Gromov P: High-resolution two-dimensional gel electrophoresis and protein identification using western blotting and ECL detection. EXS 88: 55-67, 2000.

23. Alaiya AA, Franzén B, Fujioka K, Moberger B, Schedvins K, Silfversvärd C, Linder S and Auer G: Phenotypic analysis of ovarian carcinoma: polypeptide expression in benign, borderline and malignant tumors. Int J Cancer 73: 678-683, 1997.

24. Alaiya AA, Franzén B, Moberger B, Silfverswärd C, Linder S and Auer G: Two-dimensional gel analysis of protein expression in ovarian tumors shows a low degree of intratumoral heterogeneity. Electrophoresis 20: 1039-1046, 1999.

25. Erler BS, Hsu L, Truong HM, Petrovic LM, Kim SS, Huh MH, Ferrell LD, Thung SN, Geller SA and Marchevsky AM: Image analysis and diagnostic classification of hepatocellular carcinoma using neural networks and multivariate discriminant functions. Lab Invest 71: 446-451, 1994.

26. Goldschmidt D, Decaestecker C, Berthe JV, Gordower L, Remmelink M, Danguy A, Pasteels JL, Salmon I and Kiss R: The contribution of image cytometry and artificial intelligencerelated methods of numerical data analysis for adipose tumor histopathologic classification. Lab Invest 75: 295-306, 1996.

27. Ravdin PM, Clark GM, Hilsenbeck SG, Owens MA, Vendely P, Pandian MR and McGuire WL: A demonstration that breast cancer recurrence can be predicted by neural network analysis. Breast Cancer Res Treat 21: 47-53, 1992.
28. Tewari A and Narayan P: Novel staging tool for localized prostate cancer: A pilot study using genetic adaptive neural networks. J Urol 160: 430-436, 1998.

29. Albano F, Zagaria A, Anelli L, Coccaro N, Impera L, Minervini CF, Minervini A, Rossi AR, Tota G, Casieri P, et al: Gene expression profiling of chronic myeloid leukemia with variant $\mathrm{t}(9 ; 22)$ reveals a different signature from cases with classic translocation. Mol Cancer 12: 36, 2013.

30. Verhaak RG, Wouters BJ, Erpelinck CA, Abbas S, Beverloo HB, Lugthart S, Löwenberg B, Delwel R and Valk PJ: Prediction of molecular subtypes in acute myeloid leukemia based on gene expression profiling. Haematologica 94: 131-134, 2009.

31. Alsagaby SA, Khanna S, Hart KW, Pratt G, Fegan C, Pepper C, Brewis IA and Brennan P: Proteomics-based strategies to identify proteins relevant to chronic lymphocytic leukemia. J Proteome Res 13: 5051-5062, 2014.

32. Pizzatti L, Panis C, Lemos G, Rocha M, Cecchini R, Souza GH and Abdelhay E: Label-free MSE proteomic analysis of chronic myeloid leukemia bone marrow plasma: Disclosing new insights from therapy resistance. Proteomics 12: 2618-2631, 2012.

33. Gjertsen BT and Wiig H: Investigation of therapy resistance mechanisms in myeloid leukemia by protein profiling of bone marrow extracellular fluid. Expert Rev Proteomics 9: 595-598, 2012.

34. Linger RM, Keating AK, Earp HS and Graham DK: TAM receptor tyrosine kinases: Biologic functions, signaling, and potential therapeutic targeting in human cancer. Adv Cancer Res 100: 35-83, 2008.

35. Sun WS, Fujimoto J and Tamaya T: Clinical implications of coexpression of growth arrest-specific gene 6 and receptor tyrosine kinases Axl and Sky in human uterine leiomyoma. Mol Hum Reprod 9: 701-707, 2003.

36. Zhu S, Wurdak H, Wang Y, Galkin A, Tao H, Li J, Lyssiotis CA, Yan F, Tu BP, Miraglia L, et al: A genomic screen identifies TYRO3 as a MITF regulator in melanoma. Proc Natl Acad Sci USA 106: 17025-17030, 2009.

37. Kerkhoff C, Klempt M and Sorg C: Novel insights into structure and function of MRP8 (S100A8) and MRP14 (S100A9). Biochim Biophys Acta 1448: 200-211, 1998.

38. Thomas CG, Patel RM and Bergfeld WF: Cytophagic and S-100 protein immunoreactive myeloid leukemia cutis. J Cutan Pathol 37: 390-395, 2010.

39. Ng CS, Lam TK, Chan JK, Hui PK, Ng HK, Szeto SC and Feng CS: Juvenile chronic myeloid leukemia. A malignancy of S-100 protein-positive histiocytes. Am J Clin Pathol 90: 575-582, 1988.

40. Gómez-Casares MT, García-Alegria E, López-Jorge CE, Ferrándiz N, Blanco R, Alvarez S, Vaqué JP, Bretones G, Caraballo JM, Sánchez-Bailón P, et al: MYC antagonizes the differentiation induced by imatinib in chronic myeloid leukemia cells through downregulation of $\mathrm{p} 27^{\mathrm{KIP1}}$. Oncogene 32 : 2239-2246, 2013

41. Vaqué JP, Navascues J, Shiio Y, Laiho M, Ajenjo N, Mauleon I, Matallanas D, Crespo P and León J: Myc antagonizes Ras-mediated growth arrest in leukemia cells through the inhibition of the Ras-ERK-p21 ${ }^{\text {Cipl }}$ pathway. J Biol Chem 280: 1112-1122, 2005. 